\title{
LONG-TERM OUTCOMES OF VERBAL LEARNING AND MEMORY IN SURVIVORS OF PEDIATRIC ACUTE LYMPHOBLASTIC LEUKEMIA
}

by

Blair Aronovitch

\begin{abstract}
A thesis submitted to
the Faculty of Graduate and Postdoctoral Affairs

in partial fulfillment of the requirements for the degree of

Master of Arts

in

Psychology

Carleton University

Ottawa, Ontario
\end{abstract}

C2012 Blair Aronovitch 
Library and Archives

Canada

Published Heritage

Branch

395 Wellington Street

Ottawa ON K1A ON4

Canada
Bibliothèque et

Archives Canada

Direction du

Patrimoine de l'édition

395 , rue Wellington

Ottawa ON K1A ON4

Canada
Your file Votre référence

ISBN: 978-0-494-93528-6

Our file Notre référence

ISBN: $978-0-494-93528-6$
NOTICE:

The author has granted a nonexclusive license allowing Library and Archives Canada to reproduce, publish, archive, preserve, conserve, communicate to the public by telecommunication or on the Internet, loan, distrbute and sell theses worldwide, for commercial or noncommercial purposes, in microform, paper, electronic and/or any other formats.

The author retains copyright ownership and moral rights in this thesis. Neither the thesis nor substantial extracts from it may be printed or otherwise reproduced without the author's permission.
AVIS:

L'auteur a accordé une licence non exclusive permettant à la Bibliothèque et Archives Canada de reproduire, publier, archiver, sauvegarder, conserver, transmettre au public par télécommunication ou par l'Internet, prêter, distribuer et vendre des thèses partout dans le monde, à des fins commerciales ou autres, sur support microforme, papier, électronique et/ou autres formats.

L'auteur conserve la propriété du droit d'auteur et des droits moraux qui protege cette thèse. $\mathrm{Ni}$ la thèse ni des extraits substantiels de celle-ci ne doivent être imprimés ou autrement reproduits sans son autorisation.
In compliance with the Canadian Privacy Act some supporting forms may have been removed from this thesis.

While these forms may be included in the document page count, their removal does not represent any loss of content from the thesis.
Conformément à la loi canadienne sur la protection de la vie privée, quelques formulaires secondaires ont été enlevés de cette thèse.

Bien que ces formulaires aient inclus dans la pagination, il n'y aura aucun contenu manquant. 


\begin{abstract}
Acute lymphoblastic leukemia (ALL) is the most common of all pediatric malignancies, accounting for $25 \%$ of cases annually. A wide range of treatment-related toxicities are well documented in survivors of this disease. However, little is known about long-term outcomes. This project sought to explore learning and memory performance in a cross-sectional sample of survivors and a group of healthy controls, as well in as a longitudinal sample of survivors. Overall, individuals who were treated for ALL demonstrated deficits in verbal learning and short-term memory as well as differences in the use of learning strategies on a verbal recall task. Sex, age at diagnosis, and the use of cranial radiation therapy predicted performance on the same task. These results may be useful in designing cognitive rehabilitation strategies as well as for use in classroom environments.
\end{abstract}




\section{Acknowledgements}

There are several people I would like to thank for their help with this project. Dr. Philippe Robaey: Thank you for your guidance, seemingly infinite patience, and for modeling perseverance in research. Courage! Dr. Stefania Maggi: Thank you for your advice, guidance and always providing a fresh set of eyes. Thank you to Etelle Bourassa and June Drayton Callender, for knowing the answer to every one of my million questions and for always making time for me. Mom and Dad: Thank you for all the pep

talks, the love and support, for talking it through, and modeling hard work. Dani: You are my merekat in crime, always. Thank you to Christine, Kim, and Raelyne for the late nights, early mornings, last minute edits, bottomless coffees, and countless exclamation marks. Thank you to Leah, my stats guru, for your know-how and encouragement. Finally, thank you to all the children and their families who volunteered their time to participate in this research and making this project possible. 


\section{Table of Contents}

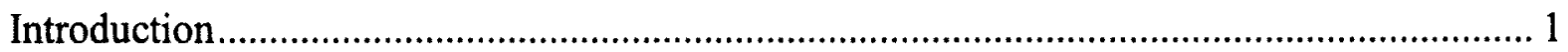

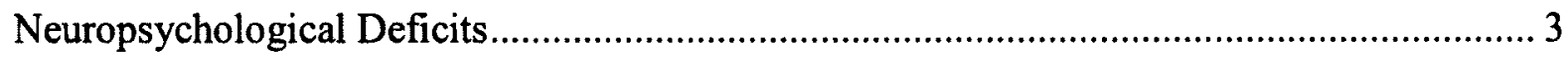

Intelligence Quotient (IQ) and Academic Achievement ..................................................... 4

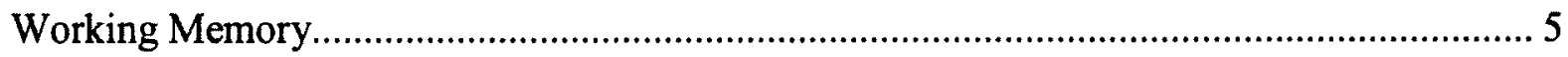

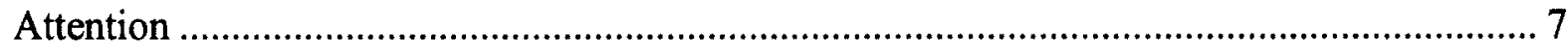

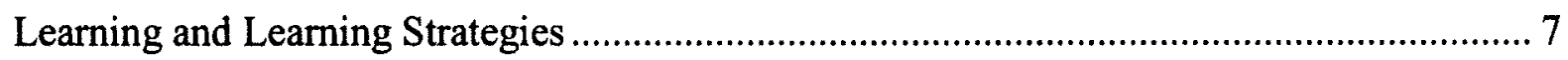

Risk Factors Associated with Differential Outcome ……….................................................. 9

Sex

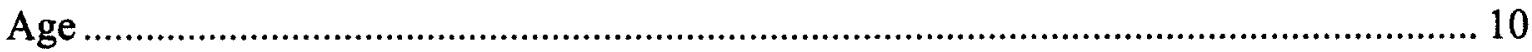

Variations in Treatment Protocol............................................................................. 11

Glucocorticoids.

Interactions Between Age, Treatment, and Sex........................................................... 15

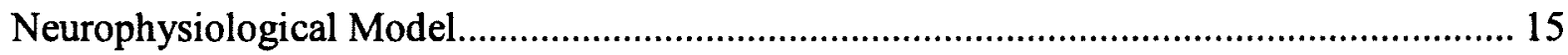

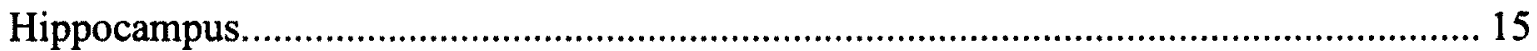

Mechanisms Behind Differential Outcomes ..................................................................... 16

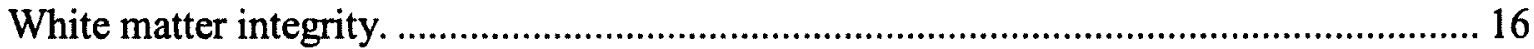

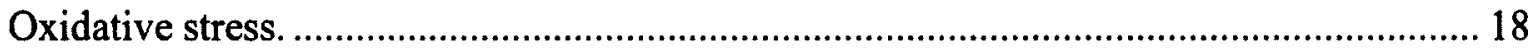

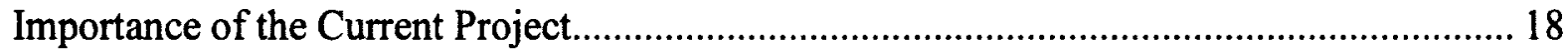

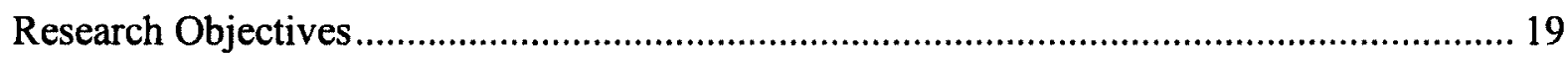

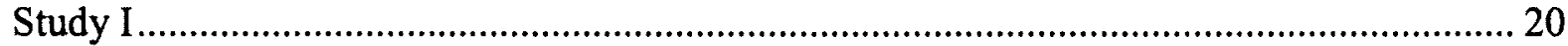

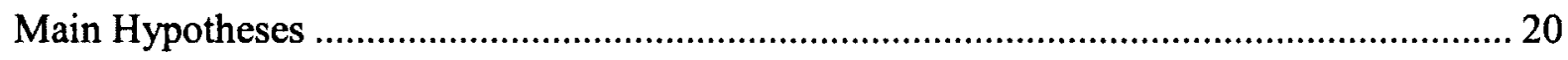

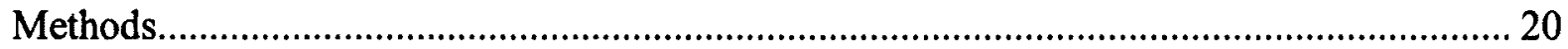


Participant Characteristics ............................................................................. 20

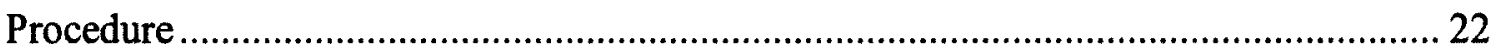

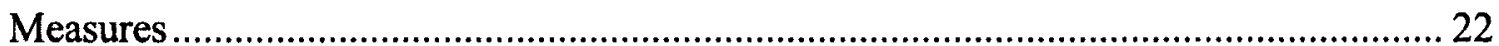

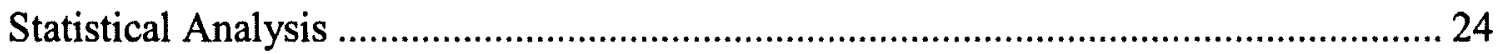

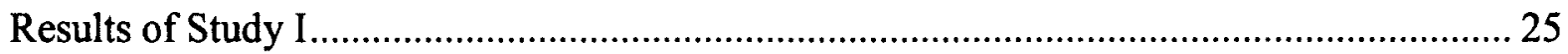

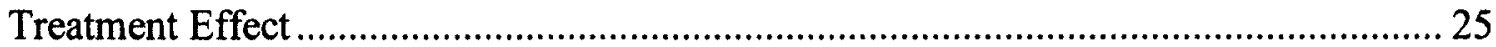

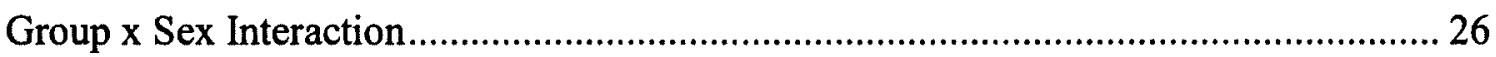

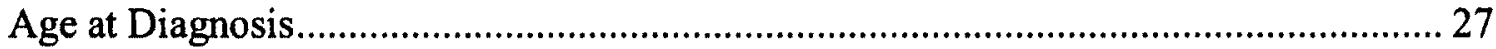

Time Elapsed Between Diagnosis and Testing ..................................................... 28

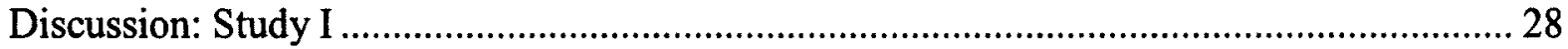

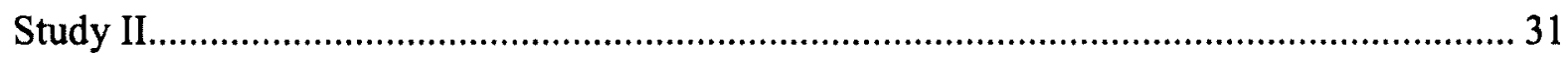

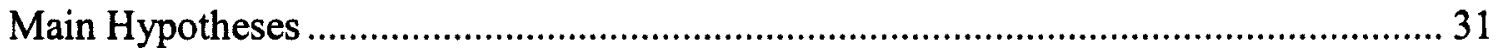

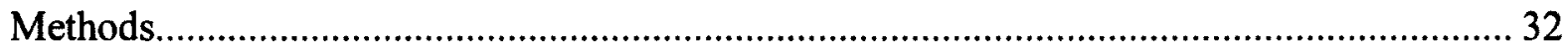

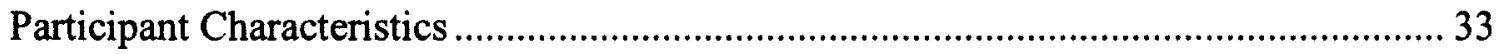

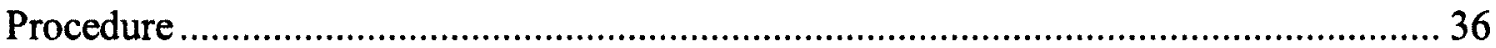

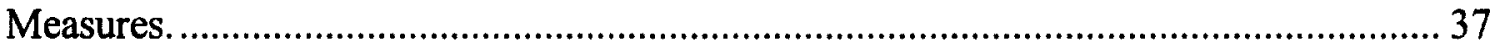

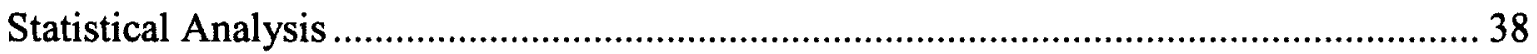

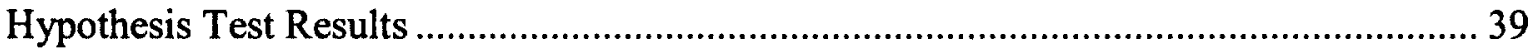

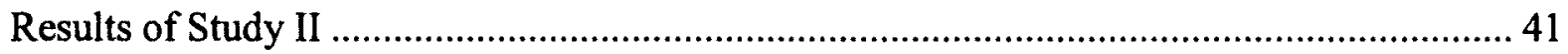

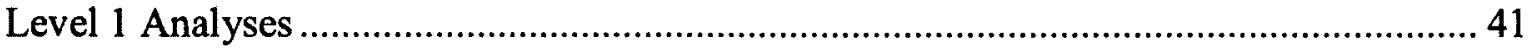

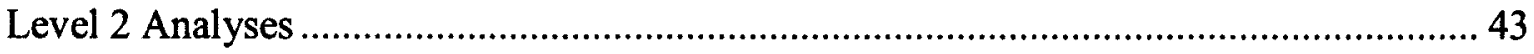

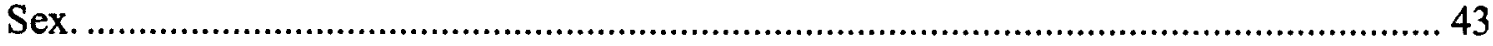

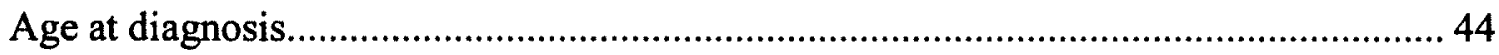




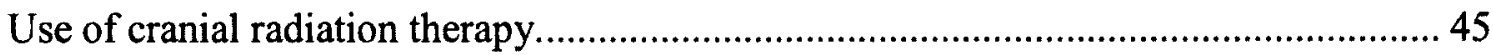

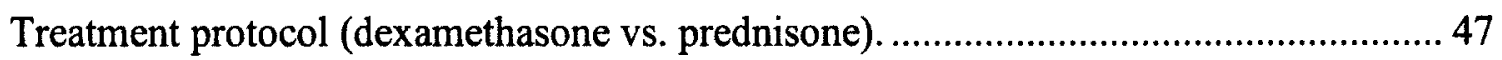

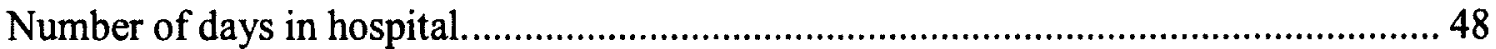

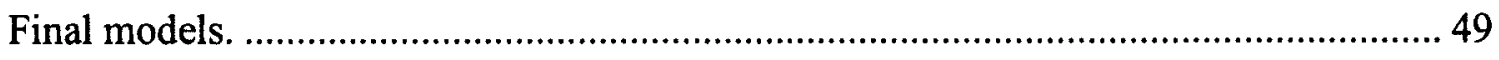

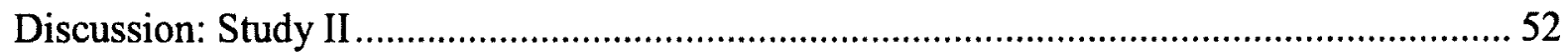

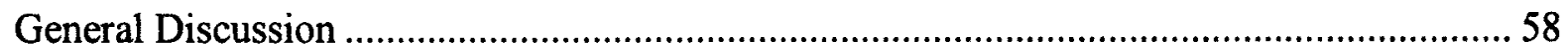

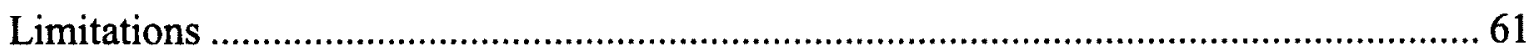

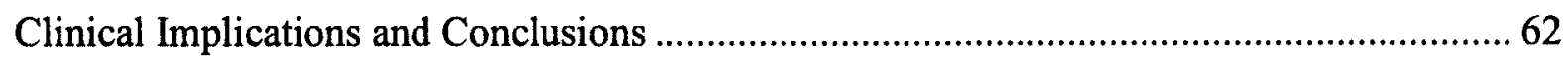

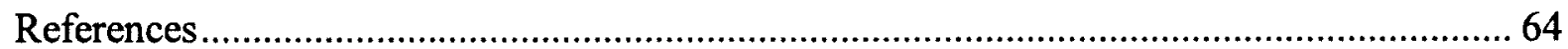




\section{List of Tables}

Table 1: Study I: Descriptive Statistics.

Table 2: Waves of Assessment 33

Table 3: Study II: Descriptive Statistics ..... 35

Table 4: DFCI Protocols .36

Table 5: Hypotheses and Multilevel Modeling Analysis. 40

Table 6: Level 1 Analyses. 42

Table 7: Level 2 Variable: Sex 43

Table 8: Level 2 Variable: Age at Diagnosis 44

Table 9: Level 2 Variable: Use of Cranial Radiation Therapy 46

Table 10: Level 2 Variable: Treatment Protocol 47

Table 11: Level 2 Variable: Number of Days in Hospital. 49

Table 12: Final Models 50

Table 13: Proactive Interference Final Model Analysis 51 


\section{List of Figures}

Figure 1: Number of Participants with Data at a Given Time Point....................................... 34

Figure 2: The Effect of Sex on List B Recall.................................................................. 44

Figure 3: The Effect of Cranial Radiation Therapy on Proactive Interference........................ 46

Figure 4: The Effect of Treatment Protocol on Proactive Interference ................................... 48 


\section{List of Appendices}

Appendix A: Study II: Research Ethics Board Approval

Appendix B: Syntax Used to Create Proactive Interference, Retroactive Interference,

and Learning Slope Variables.

Appendix C: Study II: Assessment of Linearity.

Appendix D: Study II: Assessment of Normality

Appendix E: Study II: Assessment of Normality 88 
Long-Term Outcomes of Verbal Learning and Memory in Survivors of Pediatric Acute Lymphoblastic Leukemia

Acute lymphoblastic leukemia (ALL) is the most common of all pediatric cancers, accounting for $25 \%$ of childhood malignancies (Krajinovic, Labuda, \& Sinnett, 2001). Past research has highlighted the importance of monitoring this population throughout their lifetime, as late effects stemming from cancer and cancer treatment are long-lasting (Anderson, Godber, Smibert, Weiskop, \& Ekert, 2000; Earle \& Eiser, 2007). Late cognitive effects are common, but the determinants of the lifespan trajectory of this type of deficit is unknown. Variables such as age at diagnosis, sex, and variations in treatment protocol have been identified as potential markers for variations in treatment-related toxicities (Bostrom et al., 2003; Ng et al., 2000; Waber, Gioia et al., 1990). The current project utilized crosssectional and longitudinal data sets to examine the development of deficits in verbal learning and memory and the predictive risk factors associated with differential outcomes in children treated for ALL.

Acute lymphoblastic leukemia develops when stem cells transform into lymphoblasts (leukemic cells) that restrict healthy cell and platelet growth and are unable to ward off infection. The circulatory system transports these cells to nearly every system, including the central nervous system (Pui \& Evans, 2006). The primary clinical presentation of a child with ALL varies as a function of the extent to which leukemic infiltration has occurred in the bone marrow and extramedullary sites (Stanulla \& Schrappe, 2009). Presenting symptoms typically include fever, fatigue, pallor, and bleeding (Laningham et al., 2007). Patients may be categorized as high or low risk. Patients classified as low-risk are typically between one and nine years of age with a white blood count $<50000 / \mathrm{mm}^{3}$. Individuals with a white blood 
count $>50000 / \mathrm{mm}^{3}$ and central nervous system involvement are considered high-risk, and require the most aggressive treatment protocols (Mandrell, 2009).

Data from 13 registries of the National Cancer Institute's Surveillance, Epidemiology, and End Results program revealed a modest, non-significant increase in the incidence of ALL in the United States between 1992 and 2004 (Linabery \& Ross, 2008). Currently, one to four per 100000 new cases of ALL are diagnosed each year in children 0 to 14.9 years of age (Stanulla \& Schrappe, 2009; Davidsen, Dalhoff, \& Schmiegelow, 2008) with the peak incidence at age five (Sallan, 2006).

Only 50 years ago, event-free survival rates for children diagnosed with ALL were as low as $10 \%$ (Hunger, Raetz, Loh, \& Mullighan, 2011). Dramatic improvements in survival were seen with the introduction of intrathecal or intravenous chemotherapy (chemotherapy) and cranial radiation therapy (radiation), which decreased the incidence of central nervous system involvement and relapse. Greater knowledge of prognostic features and better supportive care also contributed to improvements in survival (Cheok, Lugthart, \& Evans, 2006). Following the introduction of chemotherapy and radiation, between 1975 and 1977 the five-year survival rate for individuals with pediatric ALL increased from $57.3 \%$ to $58.9 \%$ (Ries, et al., 2008). At present, with treatment protocols continuing to improve, the five-year survival rate is reported between $80 \%$ (Pui, Relling, \& Evans, 2003) and 95\% (Plasschaert, Kamps, Vellenga, de Vries, \& de Bont, 2004).

Unfortunately, improvements in survival have been accompanied by a range of treatment-related adverse late effects. Haddy, Mosher, and Reaman (2009) conducted a longterm follow up study ( $M=5.3$ years) of 324 survivors of pediatric ALL. They reported that $74.1 \%$ of participants experienced one or more adverse events subsequent to treatment. The 
most commonly reported late effect was defective physical growth, followed by impaired neurocognitive function, emotional difficulties, cardiac abnormalities, bone morbidities, and second neoplasms. Given the high incidence of late effects, it is imperative that researchers and clinicians continue to monitor the long-term functioning of survivors of pediatric ALL.

Attempts to alleviate impairments in memory, learning, and other neurocognitive functions have included progressively lessening the intensity of treatment aimed directly at the brain by reducing doses of radiation, replacing radiation with chemotherapy, and/or excluding younger children from protocols that include the most neurotoxic treatments to the extent that these changes do not jeopardize survival rates. As a result of these strategies, the severity of neuropsychological late effects has diminished in the last decade. However, relatively large and functionally important differences in cognitive outcomes between survivors and healthy controls are still present, particularly in perceptual processing, distractibility, processing speed, verbal short-term memory, academic achievement (mathematics and reading), verbal memory, and fine motor activity (Peterson et al., 2008).

\section{Neuropsychological Deficits}

Research indicates that ALL survivors are left with more severe neuropsychological deficits than survivors of some other pediatric cancers, such as Wilms tumour (a pediatric cancer affecting the kidneys). For instance, survivors of pediatric ALL are more likely to perform poorly in primary school and have a higher incidence of problem behavior as compared to Wilms tumour survivors (Buizer, de Sonneville, van den Heuvel-Elbrink, \& Veerman, 2006). In a study by Rodgers, Britton, Morris, Kernahan, and Craft (1992) survivors of ALL performed below sibling controls and survivors of other pediatric cancers on tasks requiring strategic planning behavior related to memory functioning. Another study 
found that survivors of ALL had the most severe deficits in attention compared to a cancer control group and a healthy control group. Within the ALL group, those who received more aggressive treatment protocols experienced the most severe deficits in attention (Buizer, de Sonneville, van den Heuvel-Eibrink, \& Veerman, 2005).

Compared to their healthy counterparts, survivors of pediatric ALL have greater difficulty with tasks relating to a wide range of neuropsychological abilities. A meta-analysis by Campbell et al. (2007) found that each of the 13 mean effect sizes derived from nine neurocognitive domains (overall cognitive functioning, academic achievement, attention, executive functioning, processing speed, psychomotor skills, verbal memory, visuospatial skills, and visuospatial memory) were both negative and statistically significant. This indicates that children treated for ALL possess clinically significant deficits in these areas when compared to healthy control groups.

\section{Intelligence Quotient (IQ) and Academic Achievement}

Traditionally, global measures of abilities like IQ and academic achievement tests are used to assess cognitive outcomes. Deficits in these areas tend to emerge with time elapsed from diagnosis and treatment. A four-year follow-up study of children treated with a chemotherapy-only protocol found significant differences on full-scale IQ, verbal IQ, and performance IQ between survivors and healthy controls, accounting for age at diagnosis. Moreover, a significant group by time interaction was reported on all measures of intellectual functioning, indicating that between-group differences did not emerge until three- and fouryears post-treatment (Brown, Sawyer, Antoniou, Toogood, \& Rice, 1999).

While deficits may emerge slowly over several years, they tend to persist throughout the lifespan. A follow-up study by Lofstad, Reinfjell, Hestad, and Diseth (2009) compared a 
group of ALL survivors at 4.2 to 12.4 years post-diagnosis to an age- and sex-matched healthy control group. Total IQ scores on the Wechsler Intelligence Scale for Children Third Edition (WISC-III) fell within the normal range for $97 \%$ of participants. However, despite their normal IQ scores, a specific pattern of deficits was detected within the treatment group with significantly poorer scores on the verbal IQ, symbol search, vocabulary, similarity, and arithmetic subtests, and on the freedom from distractibility and processing speed indices.

\section{Working Memory}

Working memory refers to the temporary retention and manipulation of information that is no longer in the environment and that may be used to inform behaviour (D'Esposito \& Postle, 2002). There are several theories that attempt to explain the processes involved in working memory. D'Esposito and Postle (2002) posit that there are three elements involved in working memory: storage processes, rehearsal processes that prevent decay, and executive control processes. Executive control processes allow manipulation of storage and rehearsal, and guide the accessibility of the information (D'Esposito \& Postle, 2002). Another theory, called the working-reference memory model, asserts that in the context of learning tasks, working memory may actually be divided into two subtypes: working memory, which includes specific, personal, and contextual elements of a situation, and reference memory, which includes general information relating to rules or procedures. When learning a new task, working memory would be activated, and the emphasis would shift to reference memory once the task was learned (Kesner, 1998).

Anticancer therapy is known to negatively affect working memory and clinically meaningful impairments in working memory have been observed in survivors of pediatric 
ALL. Ashford et al. (2010) assessed attention and working memory in a sample of children treated for ALL. Participants performed below normative means on a working memory task. Further, those classified as high-risk or standard-risk also performed below normative means on measures of attention.

Working memory is also involved in memory-related phenomena such as proactive interference, which occurs when earlier learning interferes with subsequent learning. Typically with age, children are increasingly able to inhibit irrelevant information from working memory, resulting in greater capacity available for storage and executive processes (Bjorklund \& Harnishfeger, 1990). Thus, individuals who recall the most words on a task measuring span size are more likely to experience the least amount of proactive interference (Lustig, May, \& Hasher, 2001). In other words, working memory span tasks may actually assess an individual's ability to resolve interference from earlier trials while improving their performance on later trials. For individuals who have difficulty with learning (such as those treated for ALL), proactive interference may serve as an additional barrier to information retention. Increasing the efficiency of learning such that an individual may inhibit earlier learning may reduce proactive interference.

Global deficits (e.g. intelligence), may be mediated by working memory. Survivors tend to express difficulty with the arithmetic, digit span, and coding subtests of the Wechsler Intelligence Scale for Children - Revised (WISC-R; Rodgers, Horrocks, Britton, \& Kernahan, 2003). This is indicative of specific deficits in processing speed and working memory. In another study, IQ deficits were mediated by working memory deficits, though processing speed only served as a moderator of working memory deficits (Schatz, Kramer, Ablin, \& Matthay, 2000). 
Rodgers, Horrocks, Britton, and Kernahan (2003) used the Wisconsin Card Sorting Task to compare survivors of ALL with sibling controls. Individuals in the treatment group did not increase their rate of committing efficiency errors, which reflect shifting to the wrong category after receiving disconfirming feedback. However, survivors did make more distraction errors resulting from a disruption in task-maintenance set. This profile highlights that a deficit in working memory may actually underlie attention difficulties.

\section{Attention}

Attention deficits are common in survivors of pediatric ALL. Krull et al. (2011) found an association between radiation and symptoms of inattentive behavior in daily life. Based on parental report, $32 \%$ of the total sample $(n=161)$ experienced symptoms of distractibility. In addition, $22 \%$ of the sample was rated as forgetful, and $20 \%$ of parents felt that their children had difficulty organizing tasks and activities.

Deficits in attention may affect other abilities related to attention, such as information processing and reaction time. Mennes et al. (2004) reported that survivors of ALL performed significantly worse on an encoding task, a focused/divided attention task, and a memory search task compared to age- and sex-matched controls. A speed-accuracy trade-off was detected in the treatment group, where children demonstrated slower reaction times but equally accurate performance. These results indicate that survivors may process information more slowly than healthy control participants, especially when information load is increased or when attention must be focused precisely.

\section{Learning and Learning Strategies}

Anticancer therapy is detrimental to learning and learning strategies. An older study by Fogarty et al. (1988) analysed a small sample of elementary school children treated for 
ALL with radiation. Subsequent to treatment, $15 \%$ of children were diagnosed with mental retardation, $15 \%$ were diagnosed with a learning disability in reading and mathematics, and $39 \%$ were diagnosed with a learning disability in mathematics alone. Though the treatment protocol is likely outdated due to the age of the study, it is of note that difficulties with learning have been a concern for survivors of this disease for decades. A more recent review of the literature confirmed that despite the use of refined treatment protocols, deficits in learning and learning strategies persist for survivors of pediatric ALL (Bisen-Hersh, Hineline, \& Walker, 2011).

Verbal memory deficits, which are related to learning, are evident in this population. A study by Montour-Proulx et al. (2005) tracked cognitive changes in 24 children treated for ALL. The Wide Range Assessment of Memory and Learning, which includes a Verbal Memory Index (a composite score derived from five memory tasks), was used to assess memory functioning and learning. Scores on the Verbal Memory Index were more than one standard deviation below the normative mean for half the sample, indicating a specific verbal memory deficit for children treated for ALL. However, no significant differences were found for mean scores on measures of verbal and visual learning.

Precourt et al. (2002) compared verbal learning and learning strategies in females treated for ALL and healthy controls. On the California Verbal Learning Test - Children's Version (CVLT-C), patterns of learning differed between participants treated with radiation and chemotherapy, those treated on chemotherapy-only protocols, and a healthy control group. While all individuals treated for ALL were initially slower to learn than those in the control group, the rate of learning seemed to accelerate for those in the chemotherapy-only group on the last few trials. For those treated with both radiation and chemotherapy, the rate 
of learning plateaued after the third of five trials. As well, the combined chemotherapy and radiation treatment group performed more poorly on the freedom from distractibility subscore of the WISC-III, which is suggestive of an auditory-verbal learning deficit.

\section{Risk Factors Associated with Differential Outcome}

Predictive risk factors most commonly associated with differential outcomes include sex, age at diagnosis, and variations in treatment protocol.

Sex. Females tend to experience more severe adverse late effects than males subsequent to anticancer therapy. Females are more likely to develop general cognitive impairment, stunted physical growth, and to be overweight (Waber, Urion, Tarbell, \& Niemeyer, 1990). Global differences between sexes were reported in a meta-analysis by Peterson et al. (2008) where females scored significantly below males on full-scale IQ, verbal IQ, and performance IQ.

Specific patterns of neuropsychological deficits have been linked to female sex. In children treated with chemotherapy alone, performance on an arithmetic test was partly predicted by a greater difficulty appreciating the structure inherent in complex visuospatial material (as measured by the Rey-Osterrieth Complex Figure Test). Scores on the digit span subtest of the WISC-R were in turn mainly predicted by sex differences. However, reading comprehension was predicted by an index of alertness, which was unrelated to sex (Waber, Gioia et al., 1990).

Subsequent to anticancer therapy, females are particularly vulnerable to deficits in attention and working memory. A long-term follow up of pediatric ALL survivors treated with a chemotherapy-only protocol reported that while the group as a whole performed within the average range on measures of cognitive abilities and academic performance, 
females performed below normative means on the freedom from distractibility index of the WISC-III which measures attention, concentration, and working memory (Brown et al., 1999). Another study examined the relationship between sex and attention deficits in 103 survivors more than five years post-diagnosis. Females demonstrated poorer performance on the backward digit span subtest of the WISC-III, which measures working memory. However, both sexes performed below normative means on other measures of working memory (Jain, Brouwers, Okcu, Cirino, \& Krull, 2009).

Researchers have proposed several theories to explain sex differences in treatment-related toxicities. Based on research that demonstrates varying reactions to anticancer agents between males and females, some theorists assert that differences occur as a result of pharmacokinetics. Hale and Lilleyman (1991) examined treatment outcomes and toxicity in children treated with 6-mercaptopurine, an antimetabolite that inhibits DNA synthesis. Compared to males, females had more toxic reactions to the drug, but also had a better eventfree survival rate at five years. This particular difference may be related to the location of the main enzyme (hypoxanthine-guanine phosphoribosyltransferase) on chromosome $\mathrm{X}$, which is responsible for converting the inactive native 6-mercaptopurine to its cytotoxic metabolites. This hypothesis is further supported by a study in which males were found to accumulate more active metabolites of methotrexate than girls in erythrocytes when treated with the same protocol (Schmiegelow et al., 1997). Alternatively, sex differences may be related to differential development (see section on brain development to follow).

Age. The age at which a child is diagnosed (and presumably begins treatment) influences both prognosis and treatment outcomes (in relation to late effects). In a large study of children diagnosed with ALL ( $n=575)$, age was found to be a significant predictor of 
outcome. Specifically, children diagnosed at less than 12 months of age were at the greatest risk of treatment failure $(\mathrm{Ng}$, et al., 2000).

A number of studies support the hypothesis that children diagnosed at a younger age are more likely to experience difficulties in various neuropsychological domains. For instance, young age at diagnosis was identified as a risk factor in attentional deficits (Buizer, De Sonneville, Van Den Heuvel-Eibrink, Njiokiktjien, \& Veerman, 2005). Another study found that attention deficits (particularly difficulties with shifting attention) could be accounted for by treatment (especially the inclusion of radiation) early in life (Schatz, Kramer, Ablin, \& Matthay, 2004). A study by Mrakotsky et al. (2011) tracked acute neurobehavioural late effects in children treated with corticosteroid cycles every other week. Behavioural side effects increased significantly during the week of corticosteroids, but only for children less than six years of age. However, these acute effects have been found to decrease with time, reducing concerns about longer-term neurobehavioral toxicities (Marcoux, Robaey, Krajinovic, Moghrabi, \& Laverdiere, 2012).

Additionally, age-related deficits may occur as a function of hemispheric localization. Deficits in functions associated with the right hemisphere appear to be more common in children treated prior to 36 months of age. Children treated later in life tended to demonstrate deficits in functions associated with the left hemisphere (Waber, Bernstein, Kammerer, Tarbell, \& Sallan, 1992).

Variations in treatment protocol. A general trend has emerged with respect to the relationship between treatment type and treatment outcomes such that patients treated with radiation experience more severe neuropsychological late effects compared to patients treated with chemotherapy-only protocols. A longitudinal study by Harila, Winqvist, Lanning, 
Bloigu, and Harila-Saari (2009) followed 64 survivors $(n=44$ treated with radiation between 1800 and $4800 \mathrm{cGy} ; \mathrm{n}=20$ treated with chemotherapy-only) and 45 healthy controls for verbal and performance IQ, memory, orientation, attention, and motor performance 20 years post-diagnosis. Compared to controls, individuals in the treatment group demonstrated impaired memory and motor functions. A significant decline in performance IQ and verbal IQ test scores was observed in those who received radiation during the follow-up period, but only in verbal IQ for non-irradiated participants in the treatment group.

In older treatment protocols using $2400 \mathrm{cGy}$ of radiation, adverse neuropsychological effects (such as comparatively lower IQ scores) were extremely common. As a result, levels of radiation were reduced to $1800 \mathrm{cGy}$. However, survivors treated with radiation had IQ scores significantly below that of children treated with chemotherapy-only protocols. This gap in IQ has been found to worsen with the length of time elapsed from diagnosis: almost four IQ points per year since diagnosis for those who received radiation, versus no change for those who did not receive radiation (Jankovic et al., 1994).

A longitudinal study by Anderson, Godber, Smibert, Weiskop, and Ekert (2000) tracked the intellectual and educational abilities of 79 survivors of pediatric cancers including ALL, acute myeloid leukemia, non-Hodgkin's lymphoma, Wilms tumours, rhabdomyosarcoma, Ewing's tumour, and hepatoblastoma, and a group of healthy controls over time. Patients were either treated with radiation and chemotherapy or with a chemotherapy-only protocol. Significant differences were detected between treatment and control groups for all summary measures of IQ. A significant interaction was found on the digit span subtest (an index of information processing capacity) where scaled scores of the irradiated group decreased while scaled scores of the other two groups increased. No 
significant between-group differences were detected using age, sex, or socioeconomic status.

Few differences have emerged between patients on chemotherapy-only protocols. A five-year follow-up study by Spiegler et al. (2006) examined neurocognitive functioning in children treated with one of three doses of methotrexate on a chemotherapy-only protocol, or with a combination of radiation and chemotherapy. As expected, children in the combined treatment group performed more poorly on measures of visual delayed memory, verbal immediate memory, visual immediate memory, verbal delayed memory, general memory, arithmetic, reading comprehension, processing speed, attention/concentration, and on measures of global functioning. Participants in the chemotherapy-only groups scored close to the population mean on all measures except a test of impulsivity. No differences were detected between chemotherapy-only protocols.

When group differences do emerge between various chemotherapy-only protocols, they tend to be more subtle than differences detected with groups who received radiation. At 10 years post-diagnosis, Kadan-Lottick et al. (2009) assessed 171 children with ALL who had previously been randomized to receive one of two chemotherapy-only protocols (intrathecal methotrexate or triple intrathecal therapy consisting of methotrexate combined with cytarabine and hydrocortisone). Both treatment groups performed similarly on tests of full-scale intelligence quotient, academic achievement, attention/concentration, memory, and visual motor integration. They differed only slightly (one fourth of a standard deviation) on processing speed, with children in the intrathecal methotrexate group performing more poorly.

Glucocorticoids. Glucocorticoids are a fundamental element of chemotherapy treatment for pediatric ALL. Commonly used glucocorticoids include prednisone and 
dexamethasone. These drugs work by binding intracellularly to the glucocorticoid receptor and cause cell death by apoptosis (Ronghe, Burke, Lowis, \& Estlin, 2001). While glucocorticoids are highly effective anti-leukemic agents, they are also associated with major toxicities. The use of glucocortioids may be particularly deleterious to the hippocampus, resulting in specific impairments in memory (Sapolsky, 2000).

Research on treatment outcome and various glucocorticoids tends to highlight the benefit of using dexamethasone over prednisone. In a study by Mitchell et al. (2005), a large group of children $(n=1603)$ were randomly assigned to receive either dexamethasone or prednisone in the induction, consolidation, and continuation phases of treatment. At five years post-treatment, patients who received dexamethasone had a significantly better rate of event-free survival and decreased risk of isolated central nervous system relapse compared to the other groups. Bostrom et al. (2003) also found an advantage to using dexamethasone over prednisone. Patients who were randomly assigned to receive dexamethasone rather than prednisone were less likely to experience isolated central nervous system relapse and suffered fewer isolated bone marrow relapses at six years post-treatment.

In a review of the literature Inaba and Pui (2010) reported that the efficacy of prednisone and dexamethasone is dose-dependent. Up to a prednisone-to-dexamethasone dose ratio of less than seven, dexamethasone produced a greater rate of event-free survival. Further, dexamethasone was found to result in more adverse effects such as infection, osteonecrosis, and mood and behaviour problems. While glucocorticoids may enhance memory consolidation of emotionally arousing experiences, they have a profoundly detrimental effect on working memory. Studies using human participants have indicated that working memory is specifically impaired by acute doses of glucocorticoid (Lupien, Gillin, \& Hauger, 1999). 
Interactions between age, treatment, and sex. The most interesting results emerge with interactions between various predictors of treatment outcome. Lockwood, Bell, and Colegrove (1999) reported a significant interaction between treatment type (radiation vs. chemotherapy-only) and age at diagnosis on a number of sensory selection tasks. Impairments in attention were greater for children irradiated prior to 54 months of age, compared to those irradiated after 54 months of age. Attention deficits were also more pervasive for children irradiated earlier in life, with impairments detected in fundamental attention skills (e.g. focusing, simple tracking), sustained attention, and more complex attentional abilities (e.g. active mental switching, problem solving). Children who received radiation later in life primarily had difficulty with active mental switching and sustained attention.

Langer et al. (2002) looked at intelligence, memory, learning, attention, and concentration in 121 long-term survivors of ALL treated with and without radiation. A sex by treatment interaction was detected where the use of radiation was associated with lower IW scores for all participants. However, when chemotherapy was used alone, the IQ decline was worse in females than in males.

\section{Neurophysiological Model}

Hippocampus. According to the literature, working memory function is linked to the activation of various brain regions, depending on the task. In a review of $\mathrm{fMRI}$ data, D'Esposito and Postle (2002) reported that posterior brain regions underlie the storage component to working memory, and that prefrontal regions underlie the rehearsal and executive control components. However, it is the hippocampus that is most commonly cited in studies involving learning and memory. 
The hippocampal region is comprised of a number of deep brain structures (including the hippocampus, dentate gyrus, subiculum, and entorhinal cortex) that form part of the medial-temporal lobe. Information processed from all sensory modalities and association areas is first received by the entorhinal cortex where it then flows to the dentate gyrus, the hippocampus, the subiculum, and back to the entorhinal cortex before returning to the sensory areas from which it orignated. The hippocampus may also receive information through the fornix (Gluck \& Myers, 1998).

The hippocampus itself plays a key role in learning and memory. Specifically, it is thought to support short-term (information stored for seconds) and intermediate-term memory (information stores for minutes to hours), but not long-term memory (information stored for greater than a few hours; Kesner, 1998). However, the literature lacks consensus, and some researchers assert that the hippocampus is involved in long-term memory.

Studies with human participants indicate that working memory is specifically impaired by acute doses of glucocorticoids. Further, these effects are dose-dependent with greater doses resulting in greater impairments (Lupien, Gillin, \& Hauger, 1999).

Glucocorticoids are commonly administered in the continuation phase of ALL treatment and impaired memory function (and short-term memory function, in particular) often follows. High doses of glucocorticoids have been found to impair the capacity of neurons to survive various neurological insults (e.g. those that may occur with cranial radiation therapy) and these impairments are particularly pronounced in the hippocampus (Packan \& Sapolsky, 1990).

\section{Mechanisms Behind Differential Outcomes}

White matter integrity. White matter connects cortical and subcortical regions in 
functionally important ways. Namely, it plays an integral role in the transfer of information from one brain region to the next. In healthy children over five years of age, white matter correlates positively with IQ scores, particularly in frontal, occipito-parietal, and occipitotemporal-parietal areas. In other words, cognitive function correlates with greater fibre organization (Schmithorst, Wilke, Dardzinski, \& Holland, 2005).

The term leukoencephalopathy refers to changes that occur deep in white matter or subcortical gray matter and are associated with cognitive changes and learning disabilities. Radiation and chemotherapy, treatments that affect the central nervous system, are known to damage white matter tissue resulting in leukoencephalopathy.

Brain development occurs in stages, which creates periods of vulnerability. Insults to the brain are treated differently, depending on the stage of development in which they occur. Insults that occur earlier in development are assimilated into the brain and neural networks. However, insults that occur later in development are compensated by changes in cerebral structures. This causes functional, adaptive changes in the brain (Andersen, 2003). Thus, differences in neurocognitive performance may occur as a function of the age at which anticancer therapy is administered.

Sex differences in treatment-related toxicities may be related to brain morphology and maturation with respect to grey and white matter. In early development, brain regions differ in their rate of myelination and white matter tends to develop more quickly in males. This was demonstrated in an fMRI study by De Bellis et al. (2001), where a significant age by sex interaction was found in white and gray matter growth. Between 6 and 18 years of age, males experienced a significant increase in white matter (45.1\%) compared to females $(17.1 \%)$, as well as a significant reduction in gray matter volume (19.1\%) compared to females $(4.7 \%)$. In 
a secondary data analysis, Schmithorst (2009) demonstrated sex differences in the association of white matter fractional anisotropy and IQ in children ${ }^{1}$. In females between the ages of five and 18, fractional anisotropy is positively correlated with IQ and age in frontal and frontoparietal regions. However, in males fractional anisotropy is negatively correlated with IQ in those regions.

More rapid myelination in males may act as a buffer against treatment-related toxicity in tasks dependent on white matter integrity, such as those involving memory and learning. Conversely, as gray matter appears to develop more slowly for males during childhood, they may perform below females on tasks mediated by gray matter. However, this has not been demonstrated.

Oxidative stress. Oxidative stress refers to an imbalance between the production of reactive oxygen species (i.e. free radicals) and antioxidant defenses. This mechanism may underlie chemotherapy-induced central nervous system injury (El-Sabagh, Ramadan, Elslam, \& Ibrahim, 2011). Children treated at a younger age tend to have the highest levels of oxidative stress compared to older children. High levels of oxidative stress are correlated with executive dysfunction (Caron et al., 2009).

\section{Importance of the Current Project}

Given the high incidence of acute lymphoblastic leukemia, improvements in the rate of survival, and the common occurrence of treatment-related toxicities gaining a clear understanding of specific deficits, their development, and associated risk factors is of critical

${ }^{1}$ Fractional anisotropy is a measure of microstructural integrity and connectivity that reflects myelination and axonal integrity of white matter tracts (Aukema, et al., 2009). 
importance to determining the needs of this growing population. Few longitudinal studies are present in the literature and long-term health-related outcomes for this population are largely unknown. On a societal level we are faced with a growing cohort of individuals we know little about.

Specific to this project are outcomes related to verbal learning and memory. Both abilities are closely related to academic performance as well as everyday tasks. Traditional classroom environments may not encourage optimal learning for children with these types of deficits. Similarly, tasks such as recalling items off a shopping list may prove difficult for this population. These cognitive functions are specifically important from a practical standpoint, as memory plays such a central role in both the academic environment and everyday life.

This project is unique in its multi-study approach as well as in the use of multilevel modeling to investigate the long-term outcomes of verbal learning and memory in pediatric ALL survivors. While the literature is quite comprehensive with respect to detecting learning and memory impairments in survivors of ALL, no published study has used longitudinal data to examine these deficits. The use of longitudinal data will address current gaps in knowledge on the long-term outcome of these individuals.

\section{Research Objectives}

This thesis project is comprised of two secondary data analysis projects with two distinct samples of children. The first study (Study I) utilized a cross-sectional sample of school-age children with and without ALL to determine between-group differences in verbal learning and memory outcomes and identify predictive risk factors. It also sought to examine differences within the treatment group as a factor of sex, age at diagnosis, and time elapsed between diagnosis and testing. 
The second study (Study II) utilized multilevel modeling to explore treatment-related risk factors and verbal learning and memory outcomes in a longitudinal sample of children treated for ALL. The objective of this study was to understand if and how sex, age at diagnosis, the use of cranial radiation therapy, type of glucocorticoid (dexamethasone vs. prednisone) and the number of days in hospital affected the trajectory (or slope) of an individual's performance on a verbal learning and memory task over the first four years from diagnosis.

\section{Study I}

\section{Main Hypotheses}

Overall, I expected that individual- and treatment-related risk factors would account for a large proportion of between-group differences on a task of verbal learning and memory. Three predictive risk factors were expected to influence outcome for children treated for ALL:

1. Sex: Verbal learning and memory impairment would be larger in girls, as compared to boys.

2. Age at diagnosis: Verbal learning and memory impairment would be larger in children diagnosed at a younger age.

3. Delay between diagnosis and testing: Verbal learning and memory impairment would increase with time elapsed between diagnosis and assessment.

\section{Methods}

\section{Participant Characteristics}

Analysis and results from this sample have been published previously under the direction of Dr. Philippe Robaey. To date, analysis of this cross-sectional sample has resulted in one 
publication, which examined the outcomes on the California Verbal Learning Test Children's Version, the Wechsler Intelligence Scale for Children - Third Edition, and the Woodcock-Johnson Psycho-Educational Battery - Revised for the first 19 females treated for ALL (10 treated with chemotherapy only, and nine treated with a combination of chemotherapy and radiation) and 10 healthy controls enrolled in the study (Precourt et al., 2002). Results of this study demonstrated verbal learning and auditory-verbal attention deficits in females treated with cranial radiation therapy. Study I expanded on previous work by dropping the girls treated with chemotherapy only and recruiting an additional 25 male participants (between the treatment and control groups).

School-age children treated with a combination of intrathecal chemotherapy and cranial radiation therapy $(n=25)$ and healthy controls $(n=19)$ were recruited at the Ste-Justine Hospital (Montreal, Quebec) between January 1989 and December 1991. Descriptive statistics related to this sample are presented in Table 1. No group differences were detected on sex. The procedures used in this study were reviewed and approved by the Institutional Review Ethics Board at Ste-Justine Hospital. Informed consent was obtained from all parents/legal guardians and assent was obtained from the children.

Table 1

Study I: Descriptive Statistics

\begin{tabular}{lcc}
\hline & \multicolumn{2}{c}{ Group } \\
\cline { 2 - 3 } Demographic or Statistic & Treatment & Control \\
\hline Sample Size & 25 & 19 \\
Sex & & $53 \%$ \\
Male & $52 \%$ & $47 \%$ \\
Female & $48 \%$ & - \\
Mean Age at Diagnosis $(S D)$ & $3.44(1.79)$ & $9.21(1.19)$ \\
Mean Age at Testing $(S D)$ & $9.11(1.43)$ & \\
Cranial Radiation Therapy & & - \\
Received & $100 \%$ &
\end{tabular}


Did Not Receive

Type of Radiotherapy

Standard

Hyperfractionated

Methotrexate Dose

High

Low
$0 \%$

$76 \%$

$24 \%$

$64 \%$

$36 \%$

\section{Procedure}

Measures. The California Verbal and Learning Test - Children's Edition (CVLT-C) assesses the strategies and processes involved in learning and recalling verbal information (Delis, Kramer, Kaplan, \& Ober, 1994). It may be administered individually to children aged 5 to 16 . To assess learning and memory, the CVLT-C uses an everyday memory task: recalling items from a shopping list. The test involves multiple trials and uses two different lists of words. The lists, called the "Monday list" (List A) and the "Tuesday list" (List B) consist of 15 words that can be evenly divided into three semantic categories. In the first five trials, the experimenter reads the Monday list out loud and participants are asked to immediately recall the items orally. In the sixth trial, the experimenter reads the Tuesday list out loud and participants are asked to immediately recall the items orally. Participants are then asked to orally recall as many items as possible from the Monday list, without the experimenter repeating the list (short delay free-recall). This is followed by a cued recall trial of items on the Monday list (short delay cued-recall). After this, participants are given a 20-minute break. The final two trials consist of a free recall of the Monday list (long delay free-recall) and a cued-recall of the Monday list (long delay cued-recall).

The CVLT-C was standardized on a stratified sample of 920 children divided into three groups based on age ("young children", "preadolescents", "teenagers"). The sample ranged in age from 5 to 16 years and included 459 females and 461 males. Each of the three 
groups were equivalent on age-corrected scaled scores of the Vocabulary subtest of the Wechsler Intelligence Scale for Children-Revised (WISC-R). The normative reference group had an IQ comparable to that of the U.S. population of children. For each age group, the proportion of Caucasians, African Americans, Hispanics, and Other racial/ethnic group was similar to that of the racial/ethnic distribution of children aged 5 to 16 years outlined by the March 1988 Census from the United States. The sample was also stratified by parental education level and geographic region.

A number of variables are generated from the results of the CVLT-C. Serial clustering is the recall of words in the same order as they were presented. The CVLT-C provides three serial clustering indices: (1) observed serial cluster score (the number of times a child recalls two words consecutively that appear in that order on the stimulus list); (2) chance-expected serial cluster score; (3) ratio of observed to expected correct cluster scores.

Five variables representing the number of words correctly recalled per trial for the first five trials (Trial 1, Trial 2...Trial 5) is generated. Another variable representing the cumulative score of the first five trials of List A (Trials 1-5) is also generated.

Several indices are created for List B: (1) number of words recalled correctly; (2) a contrast measure (the scaled score on trial 1 of List A subtracted from the scaled score of the List B trial); (3) the number of shared and non-shared (between Lists A and B) category words recalled.

The CVLT-C also measures proactive and retroactive interference. There are three variables associated with proactive interference: (1) the immediate recall score on List B is lower than the immediate recall score on the first trial of List A; (2) recall of words from the shared categories on List B; (3) recall of words from List A on the List B trial (cross-trial 
intrusion). Retroactive interference is measured as the number of words recalled on the short delay recall of List A on Trial 5.

The CVLT-C was selected for its documented reliability and validity in assessing specific strategies and processes underlying verbal learning and memory. A factor analysis by Delis, Freeland, Kramer, and Kaplan (1988) of 286 healthy control participants and 113 neurological patients indicated that verbal memory consists of a set of components, rather than a single factor. These factors reflect learning strategy, acquisition rate, serial position effect, discriminability, and learning interference. Based on an analysis of the test's sensitivity to proactive and retroactive interference, construct validity was established (Kramer \& Delis, 1991).

\section{Statistical Analysis}

A descriptive analysis of the variables was run to characterize the sample. The results are summarized in Table 1. A series of analyses of variance were conducted to examine the effect of group membership, sex, age at diagnosis, time elapsed between diagnosis and testing, and their interactions on verbal learning and memory. The effects of age at diagnosis and time elapsed between diagnosis and testing were examined within the treatment group only. The following outcome variables were tested for between- and within-group differences: total number of words recalled across Trial 1 to Trial 5 ; number of words recalled in each of Trials 1 to 5 individually; total number of words recalled on List A Short Delay trial; total number of words recalled on List A Long Delay Cued recall trial; total number of words recalled on the List A Long Delay Free recall trial; use of serial associations; use of semantic associations; total number of words recalled on List B recall trial; and primacy and recency effects (proportion of words recalled from the beginning 
versus the end of List A over trials 1 to 5). Repeated measures ANOVAs were used to test effects across trials (i.e. total number of words recalled from Trial 1 to Trial 5). Two-way ANOVAs were used in all other cases.

\section{Results of Study I}

\section{Treatment Effect}

Across all participants, a learning effect was clear with List A trial repetitions $(\mathrm{F}(1,128.3)=78.79, p<.001, \varepsilon=0.746)$, with a linear $(\mathrm{F}(1,43)=198.73, p<.001)$ and a quadratic $(\mathrm{F}(1,43)=49.66, p<.001)$ trend. A strong learning effect was also evident when comparing the number of words recalled between List A Trial 1 and the Short- and Long-Delay Free-Recall Trials $(F(1,70.7)=88.80, p<.001, \varepsilon=0.822)$.

Though there was no overall group effect on the number of words recalled across the first five trials of List A, a significant group difference was detected on the number of words recalled on the long-delay trials. Overall, children in the treatment group $(\mathrm{M}=$ $10.4, \mathrm{SD}=1.92)$ recalled fewer words than those in the control group $(\mathrm{M}=11.68, \mathrm{SD}=$ 1.67) on the Long-Delay Cued Recall trial $(\mathrm{F}(1,42)=5.41, p=.025)$. Children in the treatment group $(M=10, S D=2.86)$ also recalled fewer words than children in the control group $(\mathrm{M}=11.74, \mathrm{SD}=1.88)$ on the Long-Delay Free Recall trial $(\mathrm{F}(1,40)=$ $4.96, p=.032)$

Significant differences in learning strategy were detected between groups. The groups differed in their propensity to recall words primarily from the beginning or the end of List $A(F(1,42)=11.35, p=.002)$. Children in the control group recalled more words from the beginning of the list (primacy effect; $\mathrm{M}=31.35, \mathrm{SD}=3.78$ ) compared to those in the treatment group $(\mathrm{M}=28.14, \mathrm{SD}=3.64 ;(\mathrm{F}(1,42)=8.21, p=0.006)$. 
Conversely, children in the treatment group recalled more words from the end of the list (recency effect; $\mathrm{M}=28.67, \mathrm{SD}=4.39)$ compared to those in the control group $(\mathrm{M}=$ $25.62, \mathrm{SD}=2.63 ; \mathrm{F}(1,42)=7.18, p=0.01)$.

Over the first five trials of List A, children in the treatment group recalled fewer words in the order they were presented (number of serial associations; $M=4, S D=2.58$ ), compared to those in the control group $(\mathrm{M}=6.11, \mathrm{SD}=4.35 ; \mathrm{F}(1,42)=4.02, p=.051)$. This pattern, where children in the control group $(\mathrm{M}=.68, \mathrm{SD}=.82)$ relied upon the serial association strategy more than those in the treatment group $(\mathrm{M}=.2, \mathrm{SD}=.5)$ was also observed on the List $B$ recall trial $(F(1,42)=5.87, p=0.02)$.

\section{Group x Sex Interaction}

Over the first five trials of List $\mathrm{A}$, females in the control group $(\mathrm{M}=53.8, \mathrm{SD}=5.63)$ recalled more words than males $(\mathrm{M}=50.67, \mathrm{SD}=5.57)$. However, males in the treatment group $(M=51.17, S D=6.04)$ recalled more words than females in the treatment group $(M=47.078, S D=10.01)$. This group by sex interaction approached significance $(\mathrm{F}(1,40)=2.63, p=0.11)$. When tested trial by trial, the interaction came close to the significance threshold for the last three trials of List A: Trial $3(\mathrm{~F}(1,40)=4.18, p=0.05)$, Trial $4(\mathrm{~F}(1,40)=3.51, p=0.07)$ and Trial $5(\mathrm{~F}(1,40)=3.15, p=0.08)$.

This trend appeared to be driven by differences in learning strategies, especially in serial associations for List A (Group by Sex: $\mathrm{F}(1,40)=8.07, p=0.007$ ). In the control group, females $(\mathrm{M}=8.3, \mathrm{SD}=4.5)$ were twice as likely to recall words in the order of presentation across the first five trials of List $A$, as compared to males $(M=3.67, S D=$ $2.65 ; \mathrm{F}(1,17)=7.26, p=0.015)$. This pattern was not observed in the treatment group (females: $\mathrm{M}=3.62, \mathrm{SD}=2.4$; males: $\mathrm{M}=4.42, \mathrm{SD}=2.81, \mathrm{~ns}$ ). Even after controlling for 
association by chance as a function of the total number of words recalled, using the serial cluster ratio this group by sex interaction remained significant $(\mathrm{F}(1,40)=7.13, p=$ $0.011)$. The serial cluster index was twice as large in females $(\mathrm{M}=3.68, \mathrm{SD}=1.85)$ as it was in males $(\mathrm{M}=1.67, \mathrm{SD}=1.126)$ in the control group $(\mathrm{F}(1,17)=7.98, p=0.012), \mathrm{a}$ difference that was absent in the treatment group (females: $\mathrm{M}=1.84, \mathrm{SD}=1.17$; males: $\mathrm{M}=2.01, \mathrm{SD}=1.17, \mathrm{~ns})$

A group by sex interaction in the propensity to recall words primarily in the order of presentation (serial association) was also significant on the List A Long-Delay FreeRecall trial $(\mathrm{F}(1,40)=5.01, p=0.031)$. Of those in the control group, females $(\mathrm{M}=2.9$, $\mathrm{SD}=3.35)$ relied more upon serial associations compared to males $(\mathrm{M}=.33, \mathrm{SD}=.71$; $(\mathrm{F}(1,17)=5.06, p=0.038)$. In the treatment group, females $(\mathrm{M}=.77, \mathrm{SD}=1.24)$ did not recall more serially associated words than males $(\mathrm{M}=.75, \mathrm{SD}=1.22 ; \mathrm{F}(1,40)=5.01$, ns).

No group by sex interaction was detectable using semantic indices (where words are recalled by category) in any of the first five trials of List A, in List B or in the delayed recalls of List A.

\section{Age at Diagnosis}

For the purpose of categorizing children as being diagnosed as a young age versus being diagnosed later in life, participants in the ALL group were divided into two subgroups: above or below the median age at diagnosis ( 32 months).

Over the first five trials of List A, children diagnosed prior to 32 months of age recalled fewer words, especially at Trial 2 (below median: $\mathrm{M}=7.54, \mathrm{SD}=3.48$; above median: $\mathrm{M}=11.42, \mathrm{SD}=2.15, \mathrm{t}(23)=-3.32, p=.003$ ), Trial 3 (below median: $\mathrm{M}=9.92$, 
$\mathrm{SD}=1.85$; above median: $\mathrm{M}=11.83, \mathrm{SD}=1.40 ; \mathrm{t}(23)=-2.89, p=.008)$, and Trial 5 (below median: $\mathrm{M}=11.08, \mathrm{SD}=1.61$; above median: $\mathrm{M}=12.41, \mathrm{SD}=1.83 ; \mathrm{t}(23)=$ $1.95, p=.064)$

Within the treatment group, those diagnosed at a younger age recalled more words from the end of List A (recency effect; below median: $\mathrm{M}=30.42, \mathrm{SD}=4.49$; above median: $\mathrm{M}=26.77, \mathrm{SD}=3.53 ; \mathrm{t}(23)=2.25, p=.035)$. They also tended to produce fewer serial associations across the first five trials of List $\mathrm{A}$ (below median: $\mathrm{M}=$ $3.15, \mathrm{SD}=1.73 ;$ above median: $\mathrm{M}=4.92, \mathrm{SD}=3.09, \mathrm{t}(23)=-1.78, p=.09)$ compared to children diagnosed later in life.

Children diagnosed at a younger age also recalled fewer words in the List A Long-Delay Free-Recall trial (below median age: $\mathrm{M}=8.92, \mathrm{SD}=3.12$; above median age: $\mathrm{M}=11.17, \mathrm{SD}=2.08 ; \mathrm{t}(23)=-2.10, p=.047)$. Younger children produced fewer serial associations in the List A Long-Delay Free-Recall trial (below median: $\mathrm{M}=.08$, $\mathrm{SD}=.28 ;$ above median: $\mathrm{M}=1.5, \mathrm{SD}=1.38 ; \mathrm{t}(11.8)=-3.50, p=.004)$.

\section{Time Elapsed Between Diagnosis and Testing}

When participants were divided into two groups (below or above the median time elapsed since diagnosis, i.e. 71 months), no significant differences were observed.

\section{Discussion: Study I}

This study demonstrated marked differences between children treated for acute lymphoblastic leukemia and healthy controls, as well as differences within the treatment group. A primacy effect was observed in the control group where a recency effect was observed in the treatment group. Within the treatment group, the recency effect was most pronounced in children diagnosed prior to 32 months of age. Differences were noted in 
learning strategies with the treatment group relying less upon serial associations than the control group. Within the treatment group, females used serial associations less across Trials 1 to 5, even when overall recall was controlled for. Females and children diagnosed at a younger age also used serial associations less on the Long Delay Recall trial. In the treatment group, females recalled fewer words than males but the opposite was observed in the control group. Children diagnosed prior to 32 months of age recalled fewer words across the first five trials of List A and on the Long Delay Recall Trial.

The results of this study point to difficulty with the transfer of information from short- to long-term memory. The decreased reliance upon serial associations is indicative of a short-term memory deficit. This is further supported by the significant recency effect detected in the treatment group, and particularly for those diagnosed at a younger age. The significant recency effect within the treatment group suggests that these participants had difficulty recalling items further back in memory.

Proactive interference plays a key role in the connection between span tasks and other tasks, such as learning (Lustig, May, \& Hasher, 2001). To some extent, proactive interference guides the amount of information that may be retrieved from working memory (Jonides \& Nee, 2006). While a certain amount of proactive interference is normal (Delis, Kramer, Kaplan \& Ober, 1994), the differences observed in the treatment group point to an impairment in efficiency of learning.

In a typical population, proactive interference decreases with age (Kail, 2002). This is consistent with our findings, as the differences found in children diagnosed at a younger age appear to be linked to proactive interference (i.e. the significant recency effect). The performance on the CVLT-C of children diagnosed at a younger age suggests 
that they do not "grow out of" proactive interference the way a typically developing child would.

The decreased reliance on serial associations and the pronounced recency effect observed in the treatment group points to a degree of interference that has hindered earlier learning at the most basic level. Since the CVLT-C is based on such a rudimentary task, the implications of this are important from a practical standpoint. The retrieval of a short list of words from memory is essential in countless day-to-day tasks, from remembering directions to studying for a test.

A strong group by sex interaction was detected in this sample, where females in the control group tended to outperform males in the control group, and males in the treatment group tended to outperform females in the treatment group. This finding is consistent with the literature in that females tend to have the most negative outcomes to treatment, particularly when radiation is included in the treatment protocol (Peterson et al., 2008). Specifically, these findings confirm earlier studies that reported the most severe short-term memory deficits subsequent to anticancer therapy occur in females (Brown et al., 1999; Waber, Gioia et al., 1990).

In Study I, no significant results were detected for time elapsed between diagnosis and testing. More precisely, within the sample treated for pediatric acute lymphoblastic leukemia (assessed on average of 5.9 years post-diagnosis), there was no significant difference between those with a delay ranging from six to eight years compared to those with a delay ranging from four to six years. As these survivors did show significant learning deficits compared to the control group, these deficits may have developed more rapidly during the first four years post-diagnosis than in the years following. Therefore, 
the objective of Study II was to explore how these verbal learning and memory deficits develop over the first four years post-diagnosis, and identify the predictors of these deficits. In statistical terms, this means that in Study I, time (i.e. time elapsed between diagnosis and testing) was treated as an inter-individual variable. In Study II, time was treated as an intra-individual variable to allow the exploration of change over time within the same person.

\section{Study II}

\section{Main Hypotheses}

This study was aimed at examining the predictors of individual developmental trajectories of memory and verbal learning in survivors of pediatric acute lymphoblastic leukemia. The objective of the analysis was twofold. First, to describe the trajectory of each participant's learning capacity with time elapsed since diagnosis. I predicted that in all participants, learning capacity would slightly but significantly decrease over time. At the second level of analysis, I examined heterogeneity in the trajectories over time elapsed since diagnosis, and described the relationship between predictors and the shape of each individual trajectory.

My main hypothesis was that learning capacity would decrease with time elapsed since diagnosis in children treated for ALL. Performance on working memory tasks seems to be closely related to the ability to resolve proactive interference (Chiappe, Hasher, \& Siegel, 2000; Rosen \& Engle, 1998). As proactive interference is a strong predictor of working memory performance on a span task (Whitney, Arnett, Driver, \& Budd, 2001), I expected proactive interference to increase with time elapsed since diagnosis. In turn, I predicted this would be related to decreased working memory capacity. I also predicted that the decline of 
these learning indices with time elapsed since diagnosis would be more severe in females, in children treated at a younger age, in children receiving cranial radiotherapy, in children receiving dexamethasone (as compared to prednisone) as part of their chemotherapy protocol, and/or in children who spent more time hospitalized relative to the rest of the sample. The number of days in hospital is an indicator of disease-related complications, where a greater number of days in hospital represents more complications.

In order to control for interindividual age-related differences, age-normed scores were used for all outcome variables.

Five risk factors were expected to predict changes in individual trajectories:

1. Sex: The rate of change with time elapsed since diagnosis would be larger in girls compared to boys.

2. Age at diagnosis: The rate of change with time elapsed since diagnosis would be larger in children diagnosed at a younger age.

3. Cranial radiation therapy: The rate of change with time elapsed since diagnosis would be larger in children treated with a combination of cranial radiation therapy and chemotherapy compared to those treated solely with chemotherapy.

4. Treatment protocol: The rate of change with time elapsed since diagnosis would be larger in children treated with dexamethasone (DFCI protocol 91-01) compared to those treated with prednisone (DFCI protocol 95-01).

5. Number of days in hospital during the induction phase of treatment: The rate of change with time elapsed since diagnosis would be larger in children who spent more time hospitalized. 
Results from this dataset have been previously published in two journal articles under the direction of Dr. Philippe Robaey. The first article examined the influence of genetic polymorphisms on intellectual impairment with IQ scores (Krajinovic et al., 2005). Results from this study indicated that a specific variant (NOS3 ${ }_{894}$ TT genotype) was linked to a reduction in IQ scores over time, dependent on the use of cranial radiation therapy. As well, the use of cranial radiation therapy, young age at diagnosis, and treatment protocol were associated with changes in IQ scores. The second article looked at the influence of predictive risk factors in internalized and externalized behavioural problems (Marcoux, Robaey, Krajinovic, Moghrabi, \& Laverdière, 2012). Results pointed to greater difficulty in resolving internalizing behavioural problems where they appeared alongside medical variables (e.g. high number of days in hospital). As well, the use of dexamethasone was associated with more externalizing behavioural problems.

\section{Participant Characteristics}

Patients diagnosed with pediatric ALL were recruited at the Ste-Justine Hospital (Montreal, Quebec) between 1993 and 1999. Inclusion criteria were as follows: (1) must be $\leq$ 18 years of age at diagnosis; (2) ALL must be the first cancer occurrence; and (3) must have normal or corrected to normal hearing and vision. Participants were required to be five years of age at time of testing in order to complete the CVLT-C. Table 2 illustrates waves of assessment for the sample from baseline to four years post-diagnosis. Figure 1 depicts the number of participants with data at a given time point.

Table 2

Waves of Assessment 


\begin{tabular}{|c|c|c|c|c|c|c|}
\hline $\begin{array}{l}\text { Time elapsed } \\
\text { since diagnosis }\end{array}$ & $\begin{array}{l}\text { At } \\
\text { diagnosis }\end{array}$ & $\begin{array}{l}\text { One year } \\
\text { post- } \\
\text { diagnosis }\end{array}$ & $\begin{array}{l}\text { Two years } \\
\text { post- } \\
\text { diagnosis }\end{array}$ & $\begin{array}{l}\text { Three } \\
\text { years post- } \\
\text { diagnosis }\end{array}$ & $\begin{array}{l}\text { Four years } \\
\text { post- } \\
\text { diagnosis }\end{array}$ & Total \\
\hline $\begin{array}{l}\text { Participants } \\
\text { first assessed at } \\
\text { diagnosis }\end{array}$ & $20^{\prime \prime}$ & $17^{\dagger}$ & $14^{7}$ & $15^{7}$ & $11^{\|}$ & $11^{11}$ \\
\hline $\begin{array}{l}\text { Participants } \\
\text { first assessed } \\
\text { one year post- } \\
\text { diagnosis }\end{array}$ & - & $27^{*}$ & $21^{\dagger}$ & $18^{\ddagger}$ & $20^{\ddagger}$ & $35^{\ddagger}$ \\
\hline $\begin{array}{l}\text { Participants } \\
\text { first assessed } \\
\text { two years post- } \\
\text { diagnosis }\end{array}$ & - & - & $21^{*}$ & $19^{\dagger}$ & $17^{\ddagger}$ & $49^{\ddagger}$ \\
\hline $\begin{array}{l}\text { Participants } \\
\text { first assessed } \\
\text { three years } \\
\text { post-diagnosis }\end{array}$ & - & - & - & $10^{*}$ & $8^{\dagger}$ & $65^{\dagger}$ \\
\hline $\begin{array}{l}\text { Participants } \\
\text { first assessed } \\
\text { four years post- } \\
\text { diagnosis }\end{array}$ & - & - & - & - & $23^{*}$ & $101^{*}$ \\
\hline Total & 20 & 44 & 56 & 62 & 79 & - \\
\hline
\end{tabular}

First assessment, irrespective of time elapsed since diagnosis

'Second assessment, irrespective of time elapsed since diagnosis

${ }^{ \pm}$Third assessment, irrespective of time elapsed since diagnosis

'Fourth assessment, irrespective of time elapsed since diagnosis

"Fifth assessment, irrespective of time elapsed since diagnosis

Figure 1. Number of Participants with Data at a Given Time Point

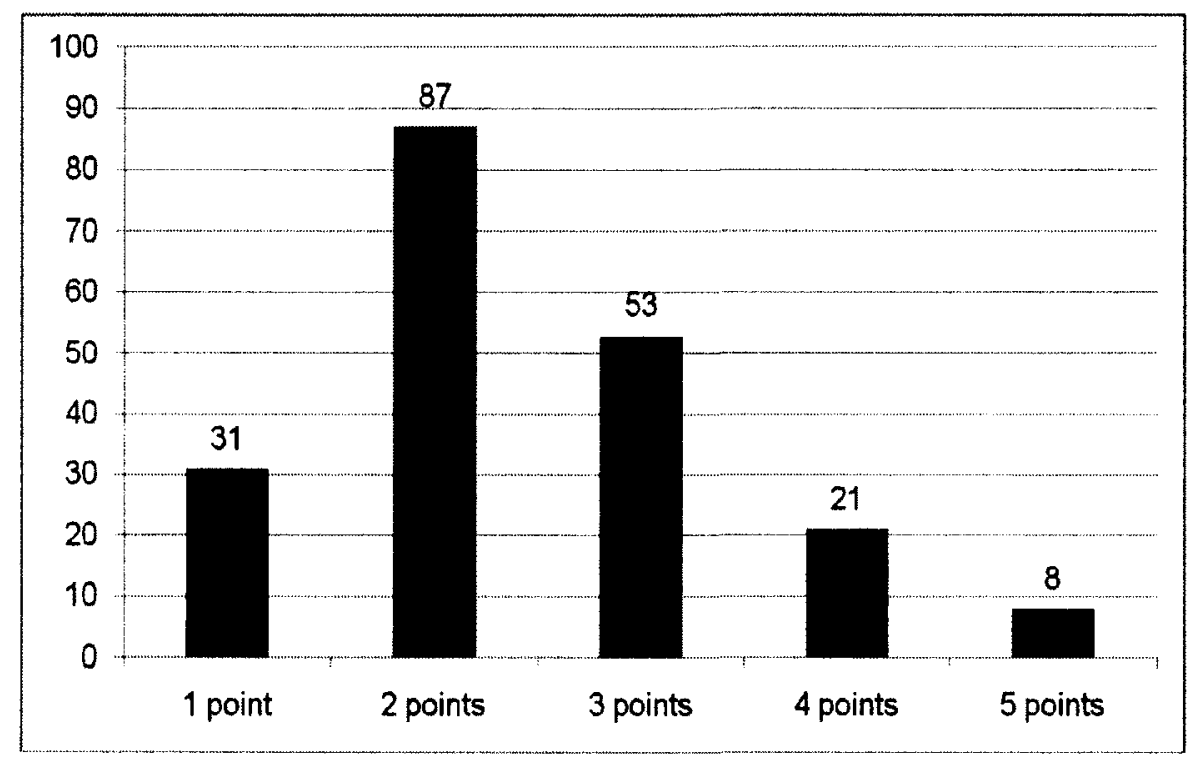


Descriptive statistics of the sample for each time point are presented in Table 3.The mean number of days in hospital was $31.02( \pm 12.01)$, with a minimum of 12 days and a maximum of 125 days. Of the total sample of 115 participants, treatment failure occurred for eight individuals. An additional seven participants refused participation.

Table 3

Study II: Descriptive Statistics

\begin{tabular}{|c|c|c|c|c|c|}
\hline & $\begin{array}{l}\text { Time 0 } \\
\text { Baseline/ } \\
\text { Diagnosis }\end{array}$ & $\begin{array}{l}\text { Time 1 } \\
\text { 1-year Post- } \\
\text { Diagnosis }\end{array}$ & $\begin{array}{l}\text { Time } 2 \\
\text { 2-years Post- } \\
\text { Diagnosis }\end{array}$ & $\begin{array}{l}\text { Time } 3 \\
\text { 3-years } \\
\text { Post- } \\
\text { Diagnosis }\end{array}$ & $\begin{array}{l}\text { Time } 4 \\
\text { 4-years Post- } \\
\text { Diagnosis }\end{array}$ \\
\hline $\operatorname{Sex}^{1}$ & $\begin{array}{l}\mathrm{M}=9 \\
\mathrm{~F}=11\end{array}$ & $\begin{array}{l}\mathrm{M}=27 \\
\mathrm{~F}=17\end{array}$ & $\begin{array}{l}\mathrm{M}=29 \\
\mathrm{~F}=27\end{array}$ & $\begin{array}{l}M=35 \\
F=27\end{array}$ & $\begin{array}{l}M=43 \\
F=36\end{array}$ \\
\hline $\begin{array}{l}\text { Mean age } \\
\text { at } \\
\text { diagnosis } \\
\text { (years) }\end{array}$ & $5.82( \pm 4.13)$ & - & - & - & - \\
\hline $\begin{array}{l}\text { Min.-max. } \\
\text { age at } \\
\text { diagnosis }\end{array}$ & $0-17$ & - & - & - & - \\
\hline $\begin{array}{l}\text { Mean age } \\
\text { at testing } \\
\text { (years) }\end{array}$ & $11.41( \pm 3.22)$ & $11.16( \pm 3.69)$ & $10.88( \pm 3.92)$ & $10.97( \pm 3.47)$ & $11.24( \pm 3.84)$ \\
\hline $\begin{array}{l}\text { Min.-max. } \\
\text { age at } \\
\text { testing }\end{array}$ & $7.66-18.08$ & $6.66-19.25$ & $6.33-20.33$ & $6.33-21.16$ & $7.08-22.42$ \\
\hline Initial risk & SR: 6 & SR: 14 & SR: 20 & SR: 35 & SR: 38 \\
\hline of relapse $e^{2}$ & HR: 14 & HR: 30 & HR: 36 & HR: 27 & HR: 41 \\
\hline Cranial & Yes: 16 & Yes: 37 & Yes: 44 & Yes: 46 & Yes: 58 \\
\hline $\begin{array}{l}\text { radiation } \\
\text { therapy } \\
\text { exposure }\end{array}$ & No: 4 & No: 7 & No: 12 & No: 16 & No: 21 \\
\hline Treatment & 91-01: 1 & $91-01: 15$ & $91-01: 21$ & $91-01: 22$ & $91-01: 34$ \\
\hline protocol & $95-01: 19$ & $95-01: 29$ & $95-01: 35$ & $95-01: 40$ & $95-01: 45$ \\
\hline
\end{tabular}

Patients were treated on Dana Farber Cancer Institute (DFCI) Protocols 91-01 or 95-01. The main differences between the two protocols are summarized in Table 4 . 
Table 4

DFCI Protocols

\begin{tabular}{lcc}
\hline & \multicolumn{2}{c}{ Treatment Protocol } \\
\cline { 2 - 3 } Antileukemic Agent & $91-01$ & $95-01$ \\
\hline Corticosteroid & Dexamethasone & Prednisone \\
Asparaginase & E. Coli, PEG, or Erwinia & E. Coli, PEG, or Erwinia \\
Doxorubicin & $360 \mathrm{mg} / \mathrm{m}^{2}$ & $300 \mathrm{mg} / \mathrm{m}^{2}$ \\
Maximum dose & Per os or intravenous & - \\
6-mercaptopurine & Source: Marcoux, Robaey, Krajinovic, Moghrabi, \& Laverdière, 2012
\end{tabular}

Procedure

Data were collected at five time points: diagnosis/baseline (T0), week 52 (T1), week 104 (T2), week 156 (T3), and week 208 (T4). Data was also collected three months postdiagnosis, but due to the close proximity to the administration of radiation, the data were excluded from the analysis. Baseline assessment (T0) was scheduled a mean 17.7 days postdiagnosis (s.d. $=17.5$ days).

The procedures used in this study were reviewed and approved by the Institutional Review Ethics Board at Ste-Justine Hospital (see Appendix A). Informed consent was obtained from all parents/legal guardians and assent was obtained from the children.

Due to the ineligibility (e.g. based on age) of participants at some time points and attrition, the number of data points per participant varies between one and five. Of a possible 115,100 participants had data for one or more time points and were included in the analysis. Full maximum likelihood estimation was used for all analyses.

Missing data were estimated with imputation using the HLM 6 statistical software package (Raudenbush et al., 2011) in which missing values are replaced by a set of $m>1$ values drawn from their predictive distribution (Schafer \& Olsen, 1998). 
Measures. The California Verbal Learning Test - Children's Version was used to examine specific verbal learning and memory abilities. To control for a possible confound between age and time, age-normed scores were used for all outcome variables. Specifically, t-score equivalents of raw scores were used for the total number of words recalled across Trials 1 to 5 and standard scores were used for the number of words recalled on: List A Trial 1, List A Trial 5, List B recall, and the short-delay free recall of List A. Standardized scores were used to compute learning slope, proactive interference, and retroactive interference (see Appendix B). While the CVLT-C is intended for individuals aged 5 to 16 years of age, participants up to 18 years of age were included in the analysis in order to maximize sample size.

Psychosocial outcomes such as maternal education (Espy, et al. 2001) and socioeconomic status (Waber, Urion et al., 1990) are known to affect treatment outcome. The Family Adversity Index (FAI) was used to control for these variables. The FAI was calculated using data on structure of the familial cell (i.e. monoparental, biparental, reconstituted, or other), level of education of each parent (or of the parent living with the child), professional occupation of one or both parents, and the age of each parent at time of the child's birth. Parental occupation is quantified with a socioeconomic index developed by Blishen and McRoberts (1976), which was based on the Canadian Census of 514 occupational categories developed according to the Canadian Classification and Dictionary of Occupations.

A higher score on the FAI indicates a greater degree of family adversity. The FAI was included at level 2 with each model tested, in order to control for the variables included in the index. The FAI varies between 0 (low risk) and 1 (high risk). For structure of the 
familial cell, a score of 0 was assigned to participants living in biparental households.

Those with monoparental or reconstituted households were assigned a score of 1 . For all other variables, a score of 0 was assigned when descriptive analysis indicated that participants fell below the $25^{\text {th }}$ percentile. A score of 1 was assigned if variable scores were greater than the $25^{\text {th }}$ percentile. Since this was a random sample (and could therefore considered representative of the population) the $25^{\text {th }}$ percentile was defined within the sample.

According to the author of this index, the FAI may only be calculated when participants have data on at least four out of the seven variables (Vitaro, Tremblay, \& Gagnon, 1992). Thus, only 81 (of the possible 100) participants received an FAI score and were included in the final analysis. A descriptive analysis of the Family Adversity Index for this sample indicated a mean score of $.17( \pm .18)$. Scores ranged from zero to .75 .

\section{Statistical Analysis}

A descriptive analysis of demographic and clinical variables (e.g., age at time of diagnosis, sex, etc.) was conducted to characterize the sample. The results of this analysis are summarized in Table 2.

A multilevel modeling approach was used to analyze this data. The multilevel approach is ideal as it allows individual changes to be tracked despite missing time points, decreases the likelihood of confounding individual change with measurement error, and does not violate the assumption of independence for repeated measures (Espy, et al. 2001).

Multilevel modeling considers two aspects of longitudinal change concurrently. The level-1 component of the multilevel model (also known as the individual growth model) is 
the change expected for every member of the population. In this case, time elapsed since diagnosis served as the level- 1 component. The outcomes to be predicted by the level- 2 submodel were the individual growth parameters of the level-1 submodel. For each growth model parameter, one portion of the level 2 submodel specified the relation between individual growth parameter and predictor. Each level-2 submodel allows for stochastic variation in the individual growth parameters, as even individuals with the same value of any given predictor may vary in their individual change trajectories (Espy, et al. 2001).

There are four key assumptions of HLM: linearity, normality, homoscedasticity (that the distributions of the residuals at level-1 are normally distributed with a mean of zero and equal variance $\sigma^{2}$ ), and independence. To assess linearity, the level 1 model for each dependent variable was graphed and examined (see Appendix C). Normality was assessed via histograms with the SPSS Statistical software package (see Appendix D). Homoscedasticity was tested using the HLM 6 software package and of the five dependent variables with significance at level 1 , only total number of words recalled across Trials 1 to 5 satisfied this assumption (see Appendix E). Given the design of the study, independence was assumed.

As per my hypotheses, the predictive factors (group variables) considered were: sex, age at diagnosis, the use of cranial radiation therapy or not, treatment protocol including either dexamethasone or prednisone, and the number of days spent in hospital during the induction phase of treatment.

\section{Hypothesis Test Results}

Each of the five hypotheses of this study was associated with one of five predictive risk factors. A total of eight dependent variables derived from the CVLT-C (learning slope, proactive interference, retroactive interference, number of words recalled at Trial 
1 , number of words recalled at Trial 5, total number of words recalled across Trials 1 to 5, number of words recalled on List B, and number of words recalled in the short delay free recall trial of List A) were systematically tested against each predictive risk factor using multilevel modeling (see Table 5). To analyse change over time, time was entered at level 1. Each predictive risk factor was subsequently tested individually at level 2 . In order to control for psychosocial variables, the Family Adversity Index was also entered at level 2 with each analysis. All variables at level 1 and level 2 were grand-mean centred.

Table 5

Hypotheses and Multilevel Modeling Analysis

\begin{tabular}{|c|c|}
\hline Hypothesis & Analysis \\
\hline $\begin{array}{l}\text { 1. The rate of change with time } \\
\text { elapsed since diagnosis will be } \\
\text { larger in girls compared to boys. }\end{array}$ & $\begin{array}{l}\text { Level-1 Model } \\
\text { Outcome variable } e_{t i}=\pi_{0 i}+\pi_{l i}^{*}\left(T_{I M E_{t i}}\right) \\
+e_{t i} \\
\text { Level-2 Model } \\
\qquad \begin{aligned} \pi_{0 i}=\beta_{00}+\beta_{01} *\left(S E X_{i}\right)+\beta_{02}^{*}\left(F A I_{i}\right)+r_{0 i} \\
\pi_{l i}=\beta_{10}+\beta_{I I}^{*}\left(S E X_{i}\right)+\beta_{12}^{*}\left(F A I_{i}\right)+r_{l i}\end{aligned}\end{array}$ \\
\hline $\begin{array}{l}\text { 2. The rate of change with time } \\
\text { elapsed since diagnosis will be } \\
\text { larger in children diagnosed at a } \\
\text { younger age compared to those } \\
\text { diagnosed later in life. }\end{array}$ & $\begin{array}{l}\text { Level-1 Model } \\
\text { Outcome variable }{ }_{t i}=\pi_{0 i}+\pi_{l i}^{*}\left(T_{M M E_{t i}}\right) \\
+e_{t i} \\
\text { Level-2 Model } \\
\qquad \begin{array}{l}\pi_{0 i}=\beta_{00}+\beta_{01} *\left(\mathrm{AGE}_{i}\right)+\beta_{02} *\left(F A I_{i}\right)+r_{0 i} \\
\pi_{l i}=\beta_{10}+\beta_{l 1} *\left(\mathrm{AGE}_{i}\right)+\beta_{l 2} *\left(F A I_{i}\right)+r_{l i}\end{array}\end{array}$ \\
\hline 3. The rate of change with time & Level-1 Model \\
\hline
\end{tabular}




\begin{tabular}{|c|c|c|}
\hline & $\begin{array}{l}\text { elapsed since diagnosis will be } \\
\text { larger in those treated with cranial } \\
\text { radiation therapy in addition to } \\
\text { chemotherapy, compared to those } \\
\text { treated solely with chemotherapy. }\end{array}$ & $\begin{array}{l}\text { Outcome variable }{ }_{t i}=\pi_{0 i}+\pi_{l i}^{*}\left(\text { TIME }_{t i}\right) \\
+e_{t i} \\
\text { Level-2 Model } \\
\qquad \begin{array}{l}\pi_{0 i}=\beta_{00}+\beta_{01} *\left(\mathrm{CRT}_{i}\right)+\beta_{02} *\left(F A I_{i}\right)+r_{0 i} \\
\pi_{1 i}=\beta_{10}+\beta_{11} *\left(\mathrm{CRT}_{i}\right)+\beta_{12} *\left(F A I_{i}\right)+r_{l i}\end{array}\end{array}$ \\
\hline 4. & $\begin{array}{l}\text { The rate of change with time } \\
\text { elapsed since diagnosis will be } \\
\text { larger in those treated with } \\
\text { dexamethasone (DFCI protocol } 91 \text { - } \\
\text { 01) compared to those treated with } \\
\text { prednisone (DFCI protocol 95-01). }\end{array}$ & $\begin{array}{l}\text { Level-2 Model } \\
\qquad \begin{aligned} \pi_{0 i}=\beta_{00}+\beta_{01} *\left(\mathrm{GLUCOCORTICOID}_{i}\right) \\
+\beta_{02} *\left(F A I_{i}\right)+r_{0 i} \\
\pi_{1 i}=\beta_{10}+\beta_{11} *\left(\mathrm{GLUCOCORTICOID}_{i}\right) \\
+\beta_{12} *\left(F A I_{i}\right)+r_{l i}\end{aligned}\end{array}$ \\
\hline 5. & $\begin{array}{l}\text { The rate of change with time } \\
\text { elapsed since diagnosis will be } \\
\text { larger in those who spent more days } \\
\text { hospitalized during the induction } \\
\text { phase of treatment. }\end{array}$ & $\begin{array}{l}\text { Level-2 Model } \\
\pi_{0 i}=\beta_{00}+\beta_{01} *\left(\mathrm{HOSPITAL}_{i}\right)+\beta_{02} *\left(F A I_{i}\right) \\
+r_{0 i} \\
\pi_{1 i}=\beta_{10}+\beta_{11} *\left(\mathrm{HOSPITAL}_{i}\right)+\beta_{12} *\left(F A I_{i}\right) \\
+r_{l i}\end{array}$ \\
\hline
\end{tabular}

\section{Results of Study II}

\section{Level 1 Analyses}

At level 1, significant change over time was detected with proactive interference, List B recall, List A free recall, and total number of words recalled over Trial 1 to 5 . Over 
time, proactive interference was seen to decrease significantly. The number of words recalled across the first five trials of List A increased over the first four years postdiagnosis, while recall on List B decreased. Recall on the List A Short Delay Free Recall trial increased significantly over the first four years post-diagnosis. While learning slope did not change significantly over time, analysis at level 2 was still pursued since highly significant variability was detected in the intercept of learning slope $(p<.001)$.

Testing of level 2 variables was not pursued where there was no significance at level 1 (with the exception of learning slope, as noted above). In other words, for the remainder of the variables participants' slopes did not vary significantly from each other. The results of the level 1 analyses are presented in Table 6.

Table 6

Level 1 Analyses

\begin{tabular}{|c|c|c|c|c|c|c|c|c|c|}
\hline & & \multicolumn{3}{|c|}{ Fixed Effects } & \multicolumn{3}{|c|}{ Random Effects } & \multicolumn{2}{|c|}{ Deviance } \\
\hline \multicolumn{2}{|l|}{ Outcome } & $\beta(S E)$ & $\mathrm{t}$ & $p$ & $\sigma_{\text {base }}^{2}$ & $\sigma_{\text {time }}^{2}$ & $R^{2}$ & Base & Time \\
\hline \multirow{2}{*}{$\begin{array}{l}\text { Proactive } \\
\text { interference }\end{array}$} & Slope & $-.20(.07)$ & -3.12 & .003 & 1.32 & 1.24 & .06 & 521.86 & 507.97 \\
\hline & Intercept & $-.70(.08)$ & -9.02 & $<.001$ & 1.32 & .001 & 1.00 & - & - \\
\hline \multirow{2}{*}{$\begin{array}{l}\text { Retroactive } \\
\text { interference }\end{array}$} & Slope & $-.04(.05)$ & -.87 & .39 & .85 & .84 & .01 & 421.49 & 420.77 \\
\hline & Intercept & $.10(.16)$ & .62 & .54 & .85 & .16 & .81 & - & - \\
\hline \multirow{2}{*}{$\begin{array}{l}\text { Learning } \\
\text { slope }\end{array}$} & Slope & $.04(.03)$ & 1.21 & .23 & .30 & .29 & .03 & 323.33 & 321.61 \\
\hline & Intercept & $1.03(.05)$ & 20.64 & $<.001$ & .30 & .05 & .00 & - & - \\
\hline \multirow[t]{2}{*}{ Trial 1} & Slope & $.05(.07)$ & .82 & .42 & 1.15 & 1.05 & .09 & 568.47 & 566.77 \\
\hline & Intercept & $.41(.20)$ & 2.11 & .04 & 1.15 & .35 & .70 & - & - \\
\hline Trial 5 & Slope & $.09(.06)$ & 1.59 & .12 & .75 & .70 & .07 & 519.14 & 516.35 \\
\hline
\end{tabular}




\begin{tabular}{llllllllll} 
& Intercept & $.15(.18)$ & .83 & .41 & .75 & .51 & .32 & - & - \\
Trial 1-5 & Slope & $\mathbf{1 . 0 2 ( . 4 6 )}$ & $\mathbf{2 . 1 9}$ & $\mathbf{. 0 3}$ & $\mathbf{6 8 . 8 9}$ & $\mathbf{6 1 . 8 0}$ & .10 & $\mathbf{1 9 0 8 . 2 3}$ & $\mathbf{1 9 0 6 . 6 0}$ \\
& Intercept & $\mathbf{5 5 . 4 2 ( . 9 2 )}$ & $\mathbf{6 0 . 1 1}$ & $<.001$ & $\mathbf{6 8 . 8 9}$ & $\mathbf{5 3 . 7 2}$ & .22 & - & - \\
List A & Slope & $\mathbf{. 0 8 ( . 0 4 )}$ & $\mathbf{1 . 7 8}$ & .08 & .46 & .42 & .09 & $\mathbf{4 8 5 . 8 2}$ & $\mathbf{4 8 1 . 1 4}$ \\
short delay & & & & & & & & & \\
free recall & & & & & & & & & \\
& & & & & & & & & - \\
List B & Slope & $\mathbf{- . 1 5}(.05)$ & $-\mathbf{3 . 4 1}$ & .001 & .74 & .70 & .05 & $\mathbf{5 0 7 . 3 0}$ & $\mathbf{4 9 8 . 5 4}$ \\
recall & & & & & & & & & \\
& Intercept & $-.12(.09)$ & -1.41 & .16 & .74 & .23 & .69 & - & - \\
\hline
\end{tabular}

Significant effects are in bold

\section{Level 2 Analyses}

Sex. The first hypothesis of this study was that the rate of change with time elapsed since diagnosis would be larger in girls, as compared to boys (see Table 7). On the List B recall trial, a marginally significant effect of sex was detected where the decrease in number of words recalled per year was smaller in males as compared to females on average. Figure 2 depicts the overall decline in the number of words recalled on the List B trial for both sexes, as well as the difference between the sexes.

Table 7

Level-2 Variable: Sex

\begin{tabular}{llllllll}
\hline & & \multicolumn{3}{c}{ Fixed Effects } & \multicolumn{3}{c}{ Random Effects } \\
\hline Outcome & & $\beta(S E)$ & $\mathrm{t}$ & $p$ & $\tau_{\text {base }}^{2}$ & $\tau_{\text {sex }}^{2}$ & $R^{2}$ \\
\hline Proactive & Slope & $-.21(.13)$ & -1.62 & .11 & .002 & .005 & .00 \\
interference & & & & & & & \\
& Intercept & $-.19(.17)$ & -1.13 & .26 & .002 & .01 & .00 \\
Learning slope & Slope & $-.10(.07)$ & -1.53 & .13 & .002 & .002 & .00 \\
& Intercept & $.12(.10)$ & 1.29 & .20 & .002 & .06 & .00
\end{tabular}




\begin{tabular}{llllllll} 
List A free recall & Slope & $.01(.09)$ & .11 & .92 & .009 & .01 & .00 \\
& Intercept & $.33(.24)$ & 1.38 & .17 & .009 & .74 & .00 \\
List B recall & Slope & $-.17(.10)$ & $-\mathbf{1 . 7 9}$ & .08 & .0003 & .0002 & .33 \\
& Intercept & $-.11(.17)$ & -.64 & .52 & .0003 & .22 & .00 \\
Trial 1-5 & Slope & $-1.12(.91)$ & -1.22 & .23 & 1.72 & 1.13 & .34 \\
& Intercept & $1.29(1.90)$ & .69 & .49 & 1.72 & 53.92 & .00 \\
\hline
\end{tabular}

Significant fixed effects are in bold

Figure 2. The Effect of Sex on List B Recall

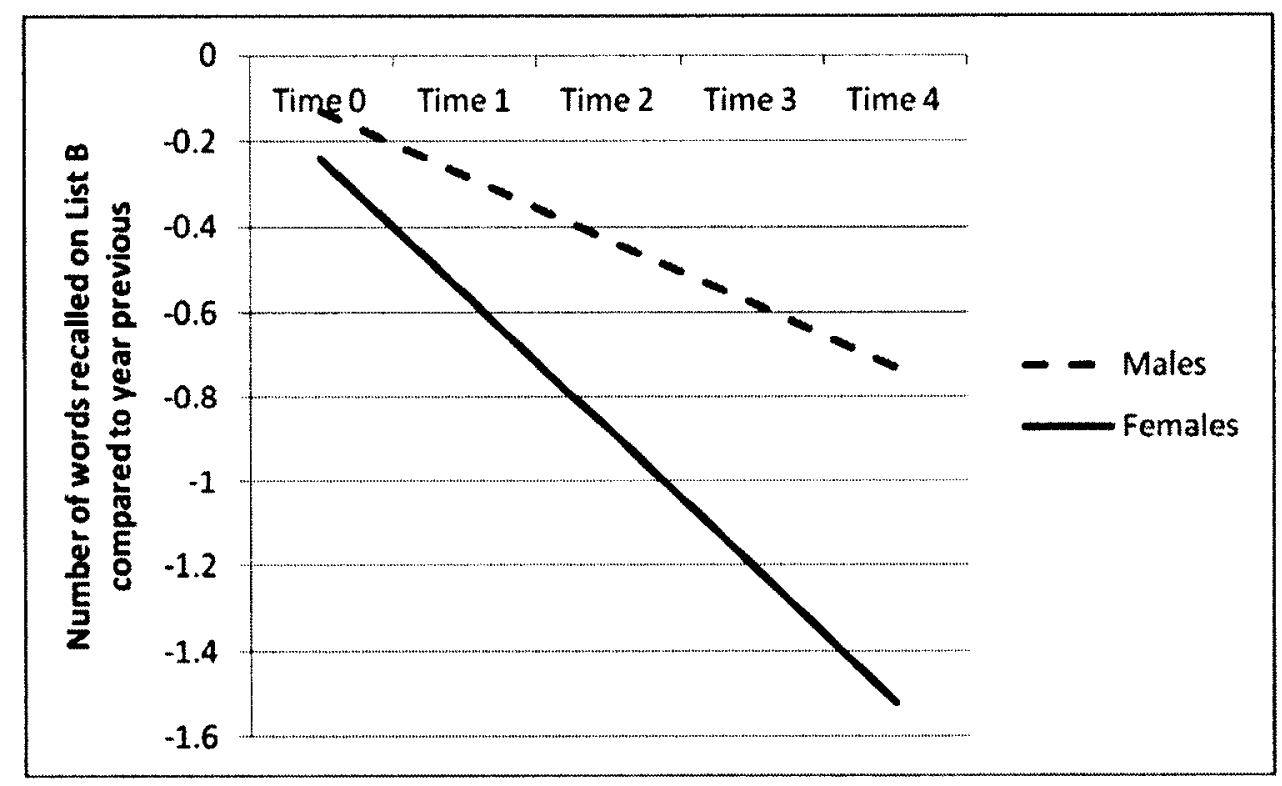

Age at diagnosis. My second hypothesis was that the rate of change with time elapsed since diagnosis would be larger in children diagnosed at a younger age compared to those diagnosed later in life (see Table 8). Age at diagnosis was entered with years as the unit of measurement. Over the first four years post-diagnosis, proactive interference significantly decreased for participants diagnosed at a younger age, relative to the sample. Additionally, a significant difference in intercept was found on proactive interference, learning slope, List A free recall, and Trial 1-5 recall.

Table 8 
Level 2 Variable: Age at Diagnosis

\begin{tabular}{|c|c|c|c|c|c|c|c|}
\hline & & \multicolumn{3}{|c|}{ Fixed Effects } & \multicolumn{2}{|c|}{ Random Effects } & \multirow[b]{2}{*}{$R^{2}$} \\
\hline Outcome & & $\beta(S E)$ & $t$ & $p$ & $\tau_{\text {base }}^{2}$ & $\tau^{2}$ age & \\
\hline \multirow{2}{*}{$\begin{array}{l}\text { Proactive } \\
\text { interference }\end{array}$} & Slope & $-.01(.02)$ &.- .34 & $<.001$ & .002 & .001 & .50 \\
\hline & Intercept & $-.09(.03)$ & -3.46 & $<.001$ & .002 & .001 & .50 \\
\hline \multirow[t]{2}{*}{ Learning slope } & Slope & $.003(.01)$ & .03 & .98 & .002 & .002 & .00 \\
\hline & Intercept & $.02(.01)$ & 1.68 & .10 & .002 & .05 & .00 \\
\hline \multirow[t]{2}{*}{ List A free recall } & Slope & $-.003(.01)$ & -.25 & .81 & .009 & .01 & .00 \\
\hline & Intercept & $.07(.03)$ & 2.57 & .01 & .009 & .69 & .00 \\
\hline \multirow[t]{2}{*}{ List $B$ recall } & Slope & $-.02(.01)$ & -1.25 & .22 & .0003 & .0002 & .33 \\
\hline & Intercept & $.04(.02)$ & 1.57 & .12 & .0003 & .21 & .00 \\
\hline \multirow[t]{2}{*}{ Trial 1-5 } & Slope & $.04(.12)$ & .31 & .76 & 1.72 & 1.82 & .00 \\
\hline & Intercept & $.51(.20)$ & 2.56 & .01 & 1.72 & 49.83 & .00 \\
\hline
\end{tabular}

Significant effects are in bold

Use of cranial radiation therapy. Thirdly, it was predicted that the rate of change with time elapsed since diagnosis would be larger in children treated with cranial radiation therapy compared to those treated with chemotherapy alone (see Table 9). Proactive interference decreased significantly over the first four years post-diagnosis for participants who received cranial radiation therapy while increasing for those who did not receive radiation. Figure 3 depicts the rate of change over time in proactive interference for irradiated and non-irradiated participants. As proactive interference is calculated by subtracting the total number of words recalled on Trial 1 of List A from the total number of words recalled on List $\mathrm{B}$, a value further from zero indicates more proactive interference (i.e. a score of -1.5 indicates more proactive interference than a score of -.7 ). In Figure 3, proactive interference is represented with absolute values for ease of 
interpretation. Over time, proactive interference increased for participants who did not receive cranial radiation therapy while it decreased for participants who received cranial radiation therapy. A significant difference in intercept was found on the List B recall trial where individuals who did not receive radiation recalled less words compared to those who did receive radiation.

Table 9

Level-2 Variable: Use of Cranial Radiation Therapy

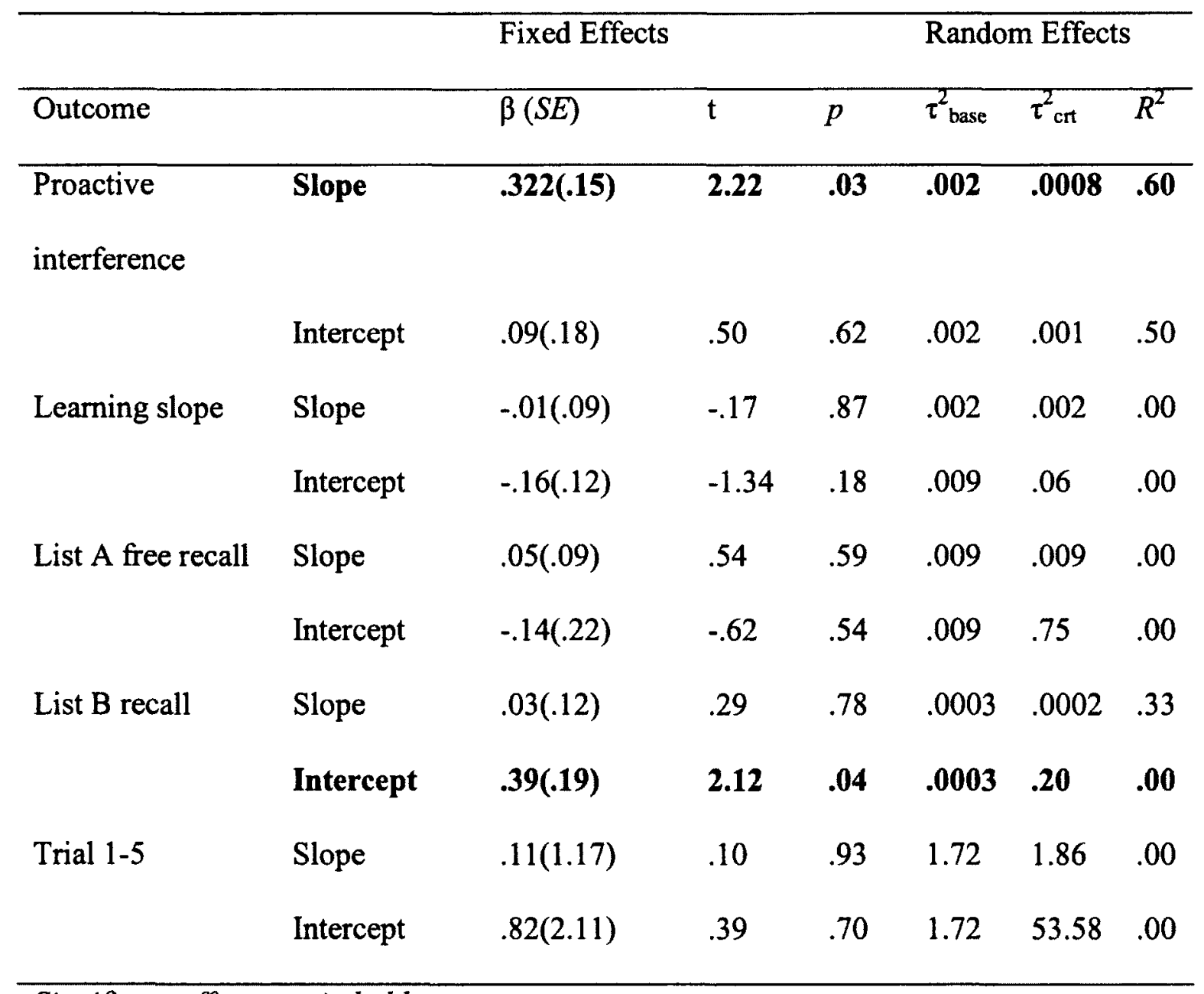

Significant effects are in bold

Figure 3. The Effect of Cranial Radiation Therapy on Proactive Interference 


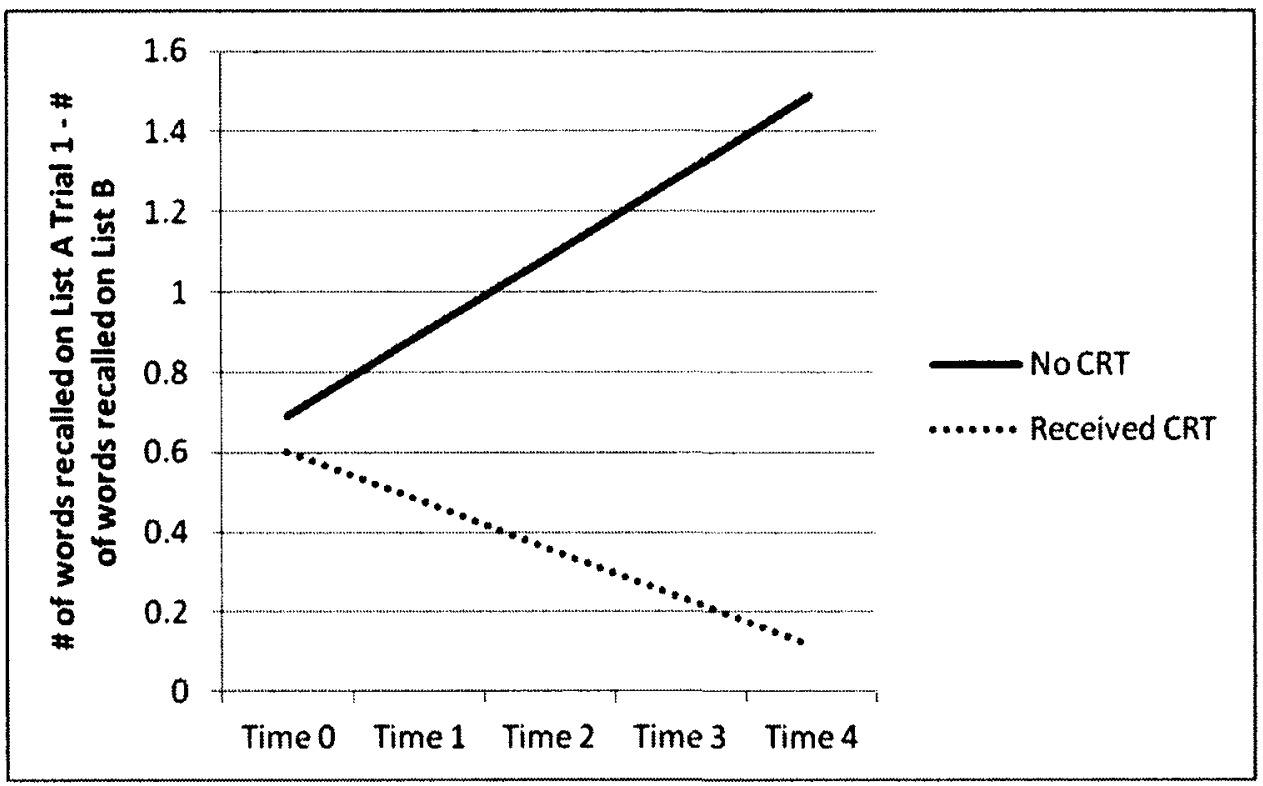

Treatment protocol (dexamethasone vs. prednisone). The fourth hypothesis of this study was that the rate of change with time elapsed since diagnosis would be larger in children treated with dexamethasone (on DFCI protocol 91-01) compared to those treated with prednisone (on DFCI protocol 95-01; see Table 10). Over the four years postdiagnosis, proactive interference increased more significantly for children treated with prednisone as compared to those treated with dexamethasone. Figure 4 shows the increase in proactive interference for all participants. Participants treated with prednisone demonstrated a slight but significantly steeper slope compared to participants treated with dexamethasone. Additionally, a significant difference in intercept was detected on learning slope with higher scores for participants who received dexamethasone compared to those treated with prednisone.

Table 10

Level-2 Variable: Treatment Protocol

\begin{tabular}{lllllll}
\hline & Fixed Effects & & \multicolumn{3}{l}{ Random Effects } \\
\hline Outcome & $\beta(S E)$ & $\mathrm{t}$ & $p$ & $\tau_{\text {base }}^{2}$ & $\tau_{\text {gluco }}^{2}$ & $R^{2}$ \\
\hline
\end{tabular}




\begin{tabular}{llllllll}
\hline Proactive & Slope & $\mathbf{0 . 0 4 ( . 1 9 )}$ & $\mathbf{- 2 . 4 0}$ & $\mathbf{. 0 2}$ & $\mathbf{. 0 0 2}$ & $\mathbf{. 0 0 2}$ & $\mathbf{. 0 0}$ \\
interference & & & & & & & \\
& Intercept & $-.05(.22)$ & -.23 & .82 & .002 & .001 & .50 \\
Learning slope & Slope & $.02(.08)$ & .21 & .83 & .002 & .003 & .00 \\
& Intercept &. $\mathbf{2 8}(.11)$ & $\mathbf{2 . 4 1}$ & .02 & .002 & .04 & .00 \\
List A free recall & Slope & $-.01(.09)$ & -.13 & .90 & .009 & .009 & .00 \\
& Intercept & $.19(.28)$ & .67 & .51 & .009 & .75 & .00 \\
List B recall & Slope & $.004(.11)$ & .03 & .97 & .0003 & .0002 & .33 \\
& Intercept & $-.24(.21)$ & -1.11 & .27 & .0003 & .21 & .00 \\
Trial 1-5 & Slope & $.05(1.05)$ & .05 & .96 & 1.72 & 1.94 & .00 \\
& Intercept & $1.55(2.11)$ & .74 & .46 & 1.72 & 52.69 & .00 \\
\hline
\end{tabular}

Significant effects are in bold

Figure 4. The Effect of Treatment Protocol on Proactive Interference

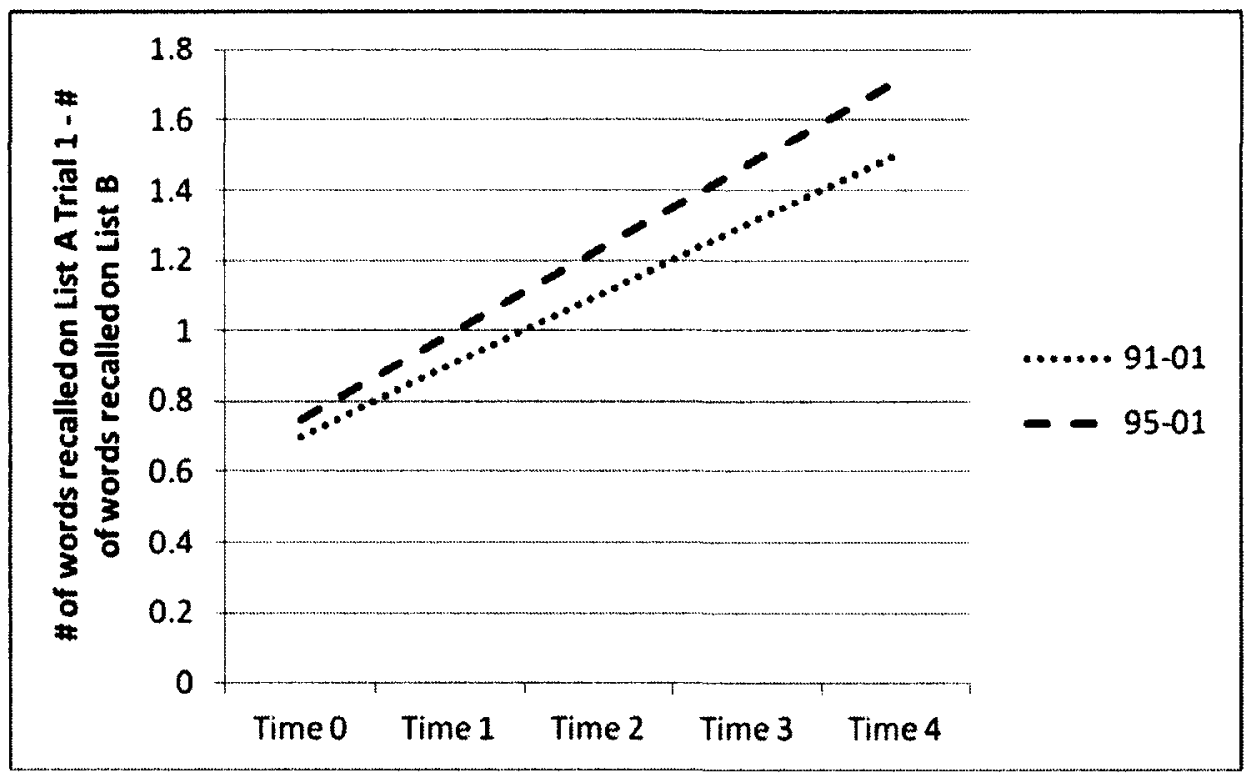

For ease of interpretation, proactive interference is represented with absolute values.

Number of days in hospital. The fifth and final hypothesis of this study was that the impairment with time elapsed since diagnosis would be larger in children who spent more time hospitalized (see Table 11). This variable was entered with days as units of measurement during the induction phase of treatment. Over the four years post-diagnosis, 
a significant decrease was observed in learning slope for participants hospitalized for a longer duration. In other words, a shorter hospital stay was associated with a greater rate of learning over time. Proactive interference decreased significantly for participants who spent less time hospitalized. As well, participants with longer hospital stays tended to recall fewer words over time on the first five trials of List A. Finally, a significant difference was detected in the intercept of the List A Short Delay Free Recall Trial where participants with longer hospital stays recalled less words on average than participants with shorter hospital stays.

Table 11

Level-2 Variable: Number of Days in Hospital

\begin{tabular}{llllllll}
\hline & & \multicolumn{5}{l}{ Fixed Effects } & \multicolumn{3}{l}{ Random Effects } \\
\hline Outcome & & $\beta(S E)$ & $\mathrm{t}$ & $p$ & $\tau_{\text {base }}^{2}$ & $\tau_{\text {hospital }}^{2}$ & $R^{2}$ \\
\hline Proactive & Slope & $-.21(.07)$ & $-\mathbf{3 . 0 1}$ & .004 & .002 & .006 & .00 \\
interference & & & & & & \\
& Intercept & $.01(.01)$ & 1.05 & .30 & .002 & .002 & .00 \\
Learning slope & Slope & $-.01(.005)$ & -2.19 & .03 & .002 & .002 & .00 \\
& Intercept & $-.001(.01)$ & -.12 & .91 & .002 & .06 & .00 \\
List A free & Slope & $-.002(.004)$ & -.57 & .57 & .009 & .01 & .00 \\
recall & & & & & & & .00 \\
& Intercept & $-.04(.02)$ & -2.01 & .05 & .009 & .66 & .00 \\
List B recall & Slope & $.0003(.01)$ & .06 & .95 & .0003 & .0002 & .33 \\
& Intercept & $-.01(.01)$ & -.53 & .60 & .0003 & .22 & .00 \\
Trial 1-5 & Slope & $-.08(.03)$ & -2.44 & .02 & 1.72 & .87 & .49 \\
& Intercept & $.02(.04)$ & .63 & .53 & 1.72 & 53.07 & .00 \\
\hline Significant fixed are in bold & & & & & &
\end{tabular}

Final models. The first part of this analysis entailed testing each predictive risk factor systematically against each outcome variable. For each of these dependent 
variables, only those predictive risk factors that were significant at level 2 were included in the final model. Table 12 displays the final model for each dependent variable.

Table 12

Final Models

\begin{tabular}{|c|c|}
\hline Dependent variable & Model \\
\hline \multirow{7}{*}{ Proactive interference } & Level-1 Model \\
\hline & $P R O A C T I V E_{t i}=\pi_{0 i}+\pi_{l i}^{*}\left(T I M E_{t i}\right)+e_{t i}$ \\
\hline & Level-2 Model \\
\hline & $\pi_{0 i}=\beta_{00}+r_{0 i}$ \\
\hline & $\pi_{I i}=\beta_{I 0}+\beta_{I I} *\left(H O S P I T A L_{i}\right)+\beta_{12}^{*}\left(C R T_{i}\right)$ \\
\hline & $+\beta_{13}{ }^{*}($ GLUCOCORTICOID $)+$ \\
\hline & $\beta_{14}^{*}\left(A G E_{i}\right)+r_{l i}$ \\
\hline \multirow{7}{*}{ Learning slope } & Level-1 Model \\
\hline & $L E A R N S L O P E_{t i}=\pi_{0 i}+\pi_{l i}^{*}\left(T I M E_{t i}\right)+e_{t i}$ \\
\hline & Level-2 Model \\
\hline & $\pi_{0 i}=\beta_{00}+\beta_{01} *\left(H O S P I T A L_{i}\right)+\beta_{02} *\left(F A I_{i}\right)+$ \\
\hline & $r_{0 i}$ \\
\hline & $\pi_{1 i}=\beta_{10}+\beta_{11}^{*}\left(\right.$ HOSPITAL $\left.L_{i}\right)+\beta_{12}^{*}\left(F A I_{i}\right)$ \\
\hline & $+r_{l i}$ \\
\hline \multirow{5}{*}{ List B recall } & Level-1 Model \\
\hline & $\operatorname{LISTB}_{t i}=\pi_{0 i}+\pi_{l i}^{*}\left(T I M E_{t i}\right)+e_{t i}$ \\
\hline & Level-2 Model \\
\hline & $\pi_{0 i}=\beta_{00}+\beta_{01} *\left(S E X_{i}\right)+\beta_{02} *\left(F A I_{i}\right)+r_{0 i}$ \\
\hline & $\pi_{1 i}=\beta_{10}+\beta_{11} *\left(S E X_{i}\right)+\beta_{12} *\left(F A I_{i}\right)+r_{1 i}$ \\
\hline \multirow{5}{*}{ Trial 1-5 } & Level-1 Model \\
\hline & $\operatorname{TRIALITOS}_{t i}=\pi_{0 i}+\pi_{l i} *\left(T I M E_{t i}\right)+e_{t i}$ \\
\hline & Level-2 Model \\
\hline & $\pi_{0 i}=\beta_{00}+\beta_{01} *\left(H O S P I T A L_{i}\right)+\beta_{02}^{*}\left(F A I_{i}\right)+$ \\
\hline & $r_{0 i}$ \\
\hline
\end{tabular}




$$
\begin{aligned}
& \pi_{1 i}=\beta_{10}+\beta_{11} *\left(\operatorname{HOSPITAL}_{i}\right)+\beta_{12} *\left(F A I_{i}\right)+ \\
& r_{l i}
\end{aligned}
$$

Only proactive interference combined multiple level 2 variables and was rerun as

a final model. The results of this analysis are summarized in Table 13.

Table 13

Proactive Interference Final Model Analysis

\begin{tabular}{lllll}
\hline & & & \multicolumn{2}{l}{ Fixed Effects } \\
\hline Outcome & & $\beta(S E)$ & $\mathrm{t}$ & $p$ \\
\hline Age at diagnosis & Slope & $-.01(.02)$ & -.30 & .77 \\
& Intercept &.$- .11(.03)$ & $-\mathbf{3 . 6 9}$ & .001 \\
Use of cranial radiation therapy & Slope & $-.27(.16)$ & -1.74 & .09 \\
Treatment protocol & Intercept &. $.44(.23)$ & $\mathbf{1 . 9 5}$ & .06 \\
& Slope & $.04(.22)$ & .18 & .86 \\
Number of days in hospital & Intercept & $.24(.26)$ & .90 & .37 \\
& Slope & $-.01(.01)$ & -.42 & .68 \\
& Intercept & $.01(.02)$ & .56 & .58 \\
\hline
\end{tabular}

Significant effects are in bold

When taken individually, age at diagnosis, use of cranial radiation therapy, treatment protocol, and number of days in hospital significantly influenced proactive interference over time. However, when all four predictive risk factors were entered into the equation together each became non-significant with the exception of use of cranial radiation therapy which remained marginally significant. In sum, none of these four predictive risk factors shares enough unique variance with proactive interference to be significant: they all act on a common source of shared variance, resulting in the individual effects becoming non-significant. However, the model as a whole was significant, and when taken individually each variable exerts significant influence over 
proactive interference scores. The results also indicate a significant difference in the intercept for age at diagnosis on proactive interference. This is not necessarily indicative of a difference in baseline and may be due to the imposition of a linear model on a process that is not strictly linear. Since I used age-corrected scores, this finding may be reflective of a difference between participants diagnosed at a younger versus older age early on.

\section{Discussion: Study II}

When taken individually, in the first four years post-diagnosis, sex of the participant, age at diagnosis, the use of cranial radiation therapy, treatment protocol, and number of days spent in hospital were identified as predictive risk factors that influenced verbal learning and memory outcomes in children treated for acute lymphoblastic leukemia, over and above the effects of time. Of the eight dependent variables tested, five were pursued with testing of level 2 variables.

When each level 2 variable was entered separately, proactive interference was significantly influenced by age at diagnosis, the use of cranial radiation therapy, treatment protocol, and number of days spent in hospital. Testing these variables separately provided only a partial view of the whole model. When taken together, only the use of cranial radiation therapy remained marginally significant. In the global model, the effect of each predictive risk factor shrunk due to shared variance with the use of cranial radiation therapy (which had a much larger effect).

These analyses also indicated that duration of hospital stay significantly influenced both learning slope and the total number of words recalled on the first five trials of List A. As well, sex significantly influenced List B recall where males tended to 
recall more words over time compared to females. number of words recalled on List B was significantly influenced by sex of the participant.

Level 1 analyses demonstrated that the number of words recalled across the first five trials of List A, as well as the number of words recalled in the List A Short Delay Free Recall trial increased over the first four years post-diagnosis. This study used agecorrected t-scores, which allowed the observation of change over years independent of age. As the scores were age-corrected, the increase in number of words recalled represented a true practice effect with the yearly repetition of the CVLT-C.

Proactive interference increased over time. This increase may be explained by the fact that overall, participants relied increasingly upon the hippocampus-dependent memory system rather than hippocampus-independent memory systems due to a disruption in transfer between systems. The increasing reliance on the hippocampusdependent memory system is related to difficulty consolidating memory in cortical networks independent on the hippocampus. The standard model of consolidation posits that the hippocampus integrates information from primary and associative cortical areas where it is encoded. With repetitive activation of the hippocampal-cortical network, cortical connections are progressively strengthened. Incremental strengthening of cortical connections eventually allows new memories to become independent of the hippocampus (McClelland, McNaughton, \& O'Reilly, 1995; Squire \& Alvarez, 1995). The process of consolidation appeared to be impaired in children treated for leukemia.

On the other hand, proactive interference decreased over time with cranial radiation therapy, age at diagnosis, treatment protocol, and hospital stay when these variables were taken individually. These results may be explained by hippocampal- 
neocortical interactions of memory formation. In typical populations, memory consolidation improves as children age (Kail, 2002), as cortical and white matter maturation contribute to the development of working memory ( $\Theta$ stby, Tamnes, Fjell, \& Walhovd, 2011). While memories mediated by the hippocampus are rich with contextual detail, as these memories become less contextually sensitive (due to the passage of time), they are retrieved independent of the hippocampus (Witigen, et al. 2010). Typically with age, children are increasingly able to inhibit irrelevant information from working memory, as storage capacity increases and executive processes (Bjorklund \& Harnishfeger, 1990). As memory becomes independent of the hippocampus, the prefrontal cortex typically assumes an integrative role via reciprocal connections with the sensory, motor, and limbic cortices. When new information matches information previously stored in cortical memory, the prefrontal cortex activity inhibits hippocampal activity to prevent encoding of redundant information by the hippocampus (Frankland \& Bontempi, 2005).

Due to the repetitive nature of the CVLT-C itself, as well as repetitions over time points, typical individuals would rely increasingly upon hippocampus-independent systems to recall this list of words. However, the negative effects of anticancer therapy on brain structures and especially on the hippocampus decreased the amount of details encoded, the duration of the hippocampal-cortical network, and ultimately the strengthening of cortical connections independent of the hippocampus. These deficits were especially severe in children who received radiation. As a consequence, consolidation as well as interference from detailed hippocampus-dependent memories on 
successive learning was impaired. These at-risk participants thus experienced less interference than participants treated at an older age with a less neurotoxic protocol.

Over the first four years post-diagnosis, proactive interference relatively decreased for participants who received cranial radiation therapy but increased for participants who were not treated with radiation. This may be indicative of less efficient learning and impaired hippocampus-dependent memory for those who received radiation. I propose that for children who received cranial radiation therapy, the hippocampusdependent learning became increasingly impaired over time, which minimized the effect of previously learned material on newly learned material. Thus, recall was relatively better in List B compared to List A Trial 1 and proactive interference decreased for these children. For children who did not receive radiation, the same hippocampal impairment was less important and thus proactive interference increased with time elapsed due to active memory of the first list of words in the hippocampal-cortical networks when learning a new list of words.

This interpretation is supported by a functional MRI study which found that during unsuccessful encoding, diffuse networks were activated in pediatric ALL survivors 20 to 30 years after treatment (Monje et al., 2012). An ineffective pattern of activation may interfere with the process required for successful encoding of memory. Four to twelve years post-treatment, ALL survivors recruited more oxygenated blood in the dorsolateral cortex and the anterior cingulate cortex during a working memory task (Robinson et al., 2010). These results suggest that the participants compensated deficits in working memory network with recruitment of a greater supply of resources (i.e. oxygen) to complete the task, though not performing at the same level of accuracy. 
Proactive interference seems to be a sensitive marker of the successful encoding of previously learned material, by measuring its interference on newly learned material. Decreased interference would then be a marker of suboptimal encoding and retrieval, as demonstrated in Study I.

Learning after cranial radiation therapy may be impaired by white matter damage, which is commonly associated with both radiation (Reddick, et al. 2005) and difficulties with learning (Roman \& Sperudo, 1995). Radiation-induced white matter damage may have decreased learning efficiency to the extent that later learning no longer affected earlier learning.

When looked at individually, proactive interference was also stronger for participants on DFCI protocol 95-01 (with prednisone) compared to those on DFCI protocol 91-1 (with dexamethasone). Hippocampus-dependent learning may have been impaired with the use of a more toxic glucocorticoid (dexamethasone) known to act directly on the hippocampus. Evidence generally indicates that dexamethasone, as compared to prednisone, has superior cerebrospinal fluid penetrance ability, potency, toxicity, withdrawal syndrome-inducing capacity, and side-effects (Stuart, Segal, \& Keady, 2005). As the effect of context depends on the function of the hippocampus and as learning is hippocampus-dependent, the effect of context may have decreased due to impairment of this structure. As a consequence, proactive interference decreased with dexamethasone use. However, when treatment protocol was entered into the final model it became non-significant. The effect of treatment protocol was reduced when the effect of cranial radiation therapy was taken into account. 
Since children who spend more time hospitalized experience more treatmentrelated complications, the number of days in hospital stood in as a proxy for treatment toxicity, including brain toxicity. When taken individually, those who spent more time in hospital may have experienced greater impairment to their hippocampus-dependent system, which in turn decreased proactive interference over time. However, when number of days in hospital was entered into the final model it became non-significant due to shared variance with cranial radiation therapy.

The effect of age at diagnosis on proactive interference was small but also negative when taken individually. With age, the difference between two $t$-scores (the score for List B minus the score for List A Trial 1) tends to become more negative. In this study, older children decreased in the number of words they called on List B compared to List A Trial 1. Thus, they experienced less proactive interference, or the increase in proactive interference over time was stronger for younger children. Conversely, younger children experienced more proactive interference over time, which is in line with greater vulnerability of the hippocampus observed in younger children. As with treatment protocol and number of days in hospital, the effect of age at diagnosis became nonsignificant when it was entered into the final model due to shared variance with cranial radiation therapy.

On average, males recalled more words on the List B trial compared to females. According to the authors of the CVLT-C, poor performance on the List B recall trial is indicative of an attention deficit and not an issue related to learning per se (Delis, Kramer, Kaplan, \& Ober, 1994). Since sex-related differences were not detected on proactive interference, it follows that this difference would be observed on List B only 
and not on List A Trial 1. Since performance on List A trials reflect learning, the relative isolation and specificity of this difference in performance strengthens the conclusion that females experience specific deficits in attention subsequent to treatment. Further, impairment in working memory may actually underlie the observed impairment in attention (Rodgers, Horrocks, Britton, \& Kernahan, 2003). This finding is consistent with the literature where females tend to be more negatively affected by anticancer therapy (Brown et al., 1998; Jain, Brouwers, Okcu, Cirino, \& Krull, 2009).

Finally, the results of this study indicated that as the number of days in hospital decreased, learning slope increased. A shorter hospital stay (and by proxy a case with less treatment-related complications) is related to steeper learning slope over time. In other words, the rate of learning over time was negatively impacted by treatment-related complications. This finding is in line with my original hypothesis. Children who experience more treatment-related complications also tend to be left with more adverse late effects.

\section{General Discussion}

The main objective of this project was to identify treatment-related risk factors of short-term memory and verbal learning outcomes in two distinct samples of children diagnosed with ALL. Study I compared a cross-sectional sample of children treated for acute lymphoblastic leukemia to a group of healthy controls on a measure of verbal learning and memory. Study II used multilevel modeling to analyze verbal learning and memory performance and its associated risk factors in a longitudinal sample of children diagnosed with ALL. The results of the first study were used to guide hypotheses of the second study. When taken together, this project addressed long-term between- and within-group differences 
in this population.

In Study I, at an average of six years post-diagnosis, ALL survivors demonstrated differences in learning strategies that point to deficits in short-term memory processes essential for successful memory encoding. Children treated for leukemia recalled more words from the end of the list (recency effect) while healthy children recalled more words from the beginning of the list (primacy effect). Similarly, children in the treatment group were less able to use serial associations as a learning strategy than those in the healthy control group. Moreover, younger age at diagnosis and female sex were clear risk factors for these differences.

Study II demonstrated that these deficits develop over time, and are detectable during the first four years post-diagnosis. Proactive interference appeared to be a sensitive marker of unsuccessful memory encoding, and revealed that in addition to younger age at diagnosis and female sex, cranial radiation therapy and the use of dexamethasone also increase the risk of developing deficits.

These results are in line with brain imaging studies that have examined brain insults in ALL survivors. Studies using MRI diffusion tensor imaging (DTI) provide a sensitive and specific index of white matter integrity. A number of DTI studies have found decreased fractional anisotropy in adult survivors of ALL associated with deficits in general intelligence, as well as more specific deficits in learning and memory (Dellani, et al. 2008; Porto, et al. 2008). In a study of ALL survivors, Khong et al. (2006) found that fractional anisotropy was significantly correlated with full-scale IQ, while controlling for age at the start of treatment, dosage of cranial radiation therapy, and the delay between treatment and assessment. Moreover, compared to healthy children, those 
treated for childhood cancers have less mean white matter fractional anisotropy, which is positively correlated with processing and motor speed (Aukema, et al. 2009).

Difficulties with short-term memory and learning detected in children treated with anticancer therapy may arise as a result of damage to white matter. White matter lesions are frequently found in children treated with cranial radiation therapy (Heckl, Aschoff, \& Kunze, 2002) and white matter integrity is associated with impairments in working memory (Charlton, et al. 2006).

Working memory performance is linked to a widespread network of specific pathways throughout the brain, specifically through the cingulum, thalamus, and white matter of the frontal and temporal lobes (Charlton, Barrick, Lawes, Markus, \& Morris, 2010). A DTI study with healthy adults reported a positive correlation between verbal working memory (on a letter span task) and white matter volume in the frontoparietal regions, as well as in a region adjacent to the right precuneus, though no significant correlations with fractional anisotropy were observed in the frontal regions (Takeuchi, et al. 2011). Neurocognitive impairment related to white matter damage may occur as a result of disruption in synchronicity between cortical regions.

The results of this project indicated sex differences, with females demonstrating more impaired performance in both studies. Sex-related differences may occur as a function of white matter development where white matter develops more quickly in males as compared to females (De Bellis, et al. 2001). Increased white matter volume may act as a buffer against treatment-related toxicity in tasks dependent on white matter integrity, such as those involving short-term memory and learning. 
Sex differences may occur as a function of phases of brain development and timing of treatment. Insults to the brain are treated differently, depending on the stage of development in which they occur. Damage to the brain that occurs earlier in life is assimilated into functional networks, whereas insults that occur much later in life are compensated for with functional adaptive changes (Andersen, 2003). Developmental processes occur in phases, setting the stage for potential periods of vulnerability.

Generally, females precede males in these developmental phases (synaptic overproduction, myelination, pruning, etc.), setting the stage for an earlier window of vulnerability (Brenhouse \& Andersen, 2011). An injury occurring at the same age may be treated differently as a function of sex. For instance, an injury occurring at an early age may be assimilated into aberrant innervation patterns in the female brain such that the functions of these abnormal networks are impaired. In the male brain, the same injury would have only a minimal effect since the process would have yet to develope or is not at its peak of vulnerability.

For acute lymphoblastic leukemia, the peak in incidence is around five years of age for males and females (Sallan, 2006). This project demonstrated that individuals diagnosed before three years of age were at increased risk of developing deficits, which may indicate a window of vulnerability where females are more at risk than males.

\section{Limitations}

This study had several limitations. Firstly, the CVLT-C was standardized on a normative population but used (for the purpose of this project) with a non-normative sample. Children treated for ALL are considered a non-normative population as they experience increased school absenteeism and elevated levels of personal and 
interpersonal stress. While the validity of the CVLT-C is well-documented in a normative population, its methodological properties are unknown in its application to this population.

A second potential limitation is that data were only available past six years on average in Study I and up to five years post-diagnosis in Study II. Deficits in short-term memory and learning are often subtle and may take several years to develop. Recently, under the co-direction of Dr. Robaey, federal funding was obtained for a 15-year followup of the same participants included in Study II. Future research will explore the same predictive risk factors in relation to neuropsychological outcomes in this sample.

The results of Study I were used to guide the hypotheses of Study II. A number of interesting results relating to learning strategies arose out of the first study. However, due to logistics it was not possible to address these findings in Study II. Age-normed data on learning strategies was not available at the time of this project and will be an area of future study.

The results of Study II must be interpreted cautiously due to issues related to data quality. Of the 100 participants included in Study II, 31 had data at only one time point. While missing data was estimated with multiple imputation, there is still some error associated with this process. Additionally, heteroscedasticity was violated for all but one of the dependent variables tested at level 2 .

A final limitation of Study II is that it lacked a control group. However, the use of a baseline measurement as well as multiple measurement times made it possible to separate the effects of anticancer therapy from the effects of time.

\section{Clinical Implications and Conclusions}


This thesis project demonstrated clear and measurable differences in short-term memory and learning in children treated for acute lymphoblastic leukemia, as well as treatment-related predictive risk factors. Many studies have highlighted the significant adverse effects of anticancer therapy on cognitive functioning. However, there is a paucity of literature on long-term effects. The results of this study highlighted between-group as well as within-group differences in children treated for ALL. Female sex, age at diagnosis, treatment protocol, the use of cranial radiation therapy, and hospital stay predicted poorer performance on a measure of verbal learning and memory.

While these results indicate comparatively weak performance on a measure of verbal learning and memory in children treated for ALL and particularly for those having the associated predictive risk factors, research indicates these differences may be reversible. An early case study reported that early identification and modifications to the chemotherapy protocol not only eliminated leukoencephalopathic sequenale, but actually reduced white matter damage (Gay, Bodensteiner, Nitschke, Sexauer, \& Wilson, 1989). Future research may explore this further, particularly in its application to specific deficits. The results of this project may be useful in guiding classroom strategies, as well as cognitive rehabilitation strategies. Increased repetitions may strengthen transfer of memories from hippocampusdependent to -independent systems. 


\section{References}

Andersen, S.L. (2003). Trajectories of brain development: point of vulnerability or window of opportunity?. Neuroscience and Biobehavioural Reviews, 27, 3-18. doi: $10.1016 / \mathrm{S} 0149-7634(03) 00005-8$

Anderson, V.A., Godber, T., Smibert, E., Weiskop, S., \& Ekert, H. (2000). Cognitive and academic outcome following cranial irradiation and chemotherapy in children: a longitudinal study. British Journal of Cancer, 82(2), 255-262.

Ashford, J., Schoffstall, C., Reddick, W.E., Leone, C., Laningham, F.H., Glass, J.O., ... Conklin, H.M. (2010). Attention and working memory abilities in children treated for acute lymphoblastic leukemia. Cancer, 116, 4638-45.

Aukema, E.J., Caan, M.W., Oudhuis, N., Majoie, C.B., Vos, F.M., Reneman, L., ... Schouten-van Meeteren, A.Y. (2009). White matter fractional anisotropy correlates with speed of processing and motor speed in young childhood cancer survivors. International Journal of Radiation, Oncology, Biology, Physics, 74(3), 837-843. doi: 10.1016/j.ijrobp.2008.08.060

Bisen-Hersh, E.B., Hineline, P.N., \& Walker, E.A. (2011). Disruption of learning processes by chemotherapeutic agents in childhood survivors of acute lymphoblastic leukemia and preclinical models. Journal of Cancer, 2, 292-301.

Bjorklund, D.F. \& Harnishfeger, K.K. (1990). The resources construct in cognitive development: diverse sources of evidence and a theory of inefficient inhibition. Developmental Review, 10, 48-71. doi: 10.1016/0273-2297(90)90004-N

Blishen, B.R. \& McRoberts, H.A. (1976). A revised socioeconomic index for occupations in Canada. Canadian Review of Sociology and Anthropology, 13, 71-79. doi: 
10.1111/j.1755-618X.1976.tb00759.x

Bostrom, B.C., Sensel, M.R., Sather, H.N., Gaynon, P.S., La, M.K.,Johnston, K., ... Trigg, M.E. (2003). Dexamethasone versus prednisone and daily oral versus weekly intravenous mercaptopurine for patients with standard-risk acute lymphoblastic leukemia: a report from the Children's Cancer Group. Blood, 101, 3809-3817. doi: 10.1182/blood-2002-08-2454.

Brenhouse, H.C. \& Andersen, S.L. (2011). Developmental trajectories during adolescence in males and females: a cross-species understanding of underlying brain changes. Neuroscience \& Biobehavioural Reviews, 35, 1687-1703.

Brown, R.T., Madan-Swain, A., Walco, G.A., Cherrick, I., Ievers, C.E., Conte, ... Lauer, S.J. (1998). Cognitive and academic late effects among children previously treated for acute lymphocytic leukemia receiving chemotherapy as CNS prophylaxis. Journal of Pediatric Psychology, 23(5), 333-340.

Brown, R.T., Sawyer, M.G., Antoniou, G., Toogood, I., \& Rice, M. (1999). Longitudinal follow-up of the intellectual and academic functioning of children receiving central nervous system-prophylactic chemotherapy for leukemia: a four-year final report. Developmental and Behavioural Pediatrics, 20(5), 373-377.

Buizer, A., de Sonneville, L.M.J., van den Heuvel-Elbrink, M.M., \& Veerman, A.J.P. (2006). Behavioural and educational limitations after chemotherapy for childhood acute lymphoblastic leukemia or Wilms tumor. Cancer, 106, 2067-2075. doi: $10.1002 /$ cncr. 21820

Buizer, A., de Sonneville, L.M.J., van den Heuvel-Eibrink, M.M., \& Veerman, A.J.P. (2005). Chemotherapy and attentional dysfunction in survivors of childhood acute 
lymphoblastic leukemia: effect of treatment intensity. Pediatric Blood Cancer, 45, 281-290. doi: 10.1002/pbc.20397

Campbell, L.K., Scaduto, M., Sharp, W., Dufton, L., Van Slyke, D., Whitlock, J.A., \& Compas, B. (2007). A meta-analysis of the neurocognitive sequelae of treatment for childhood acute lymphocytic leukemia. Pediatric Blood Cancer, 49, 65-73. doi: $10.1002 / \mathrm{pbc} .20860$

Caron, J.E., Krull, K.R., Hockenberry, M.H., Jain, N., Kaemingk, K., \& Moore, I.M. (2009). Oxidative stress and executive function in children receiving chemotherapy for acute lymphoblastic leukemia. Pediatric Blood Cancer, 53, 551-556. doi: $10.1002 / \mathrm{pbc} .22128$

Charlton, R.A., Barrick, T.R., Lawes, N.C., Markus, H.S., \& Morris, R.G. (2010). White matter pathways associated with working memory in normal aging. Cortex, 46, 474489. doi: 10.1016/j.cortex.2009.07.005

Charlton, R.A., Barrick, T.R., McIntyre, D.J., Shen, Y., O'Sullivan, M., ... Markus, H.S. (2006). White matter damage on diffusion tensor imaging correlates with age-related cognitive decline. Neurology, 66(2), 217-222. doi:

10.1212/01.wnl.0000194256.15247.83

Cheok, M.H., Lugthart, S., \& Evans, W.E. (2006). Pharmacogenomics of acute leukemia. Annual Review of Pharmacology and Toxicology, 46, 317-353. doi: 10.1146/annurev.pharmtox.45.120403.100018

Chiappe, P., Hasher, L., \& Siegel, L.S. (2000). Working memory, inhibitory control, and reading disability. Memory \& Cognition, 28, 8-17.

D'Esposito, M. \& Postle, B.R. (2002). The neural basis of working memory storage, 
rehearsal, and control processes: Evidence from patient and functional magnetic resonance imaging studies. In L.R. Squire \& D.L. Schacter (Eds.), Neuropsychology of memory ( $3^{\text {rd }}$ Ed.; pp. 215-224). New York: The Guilford Press.

Davidsen, M.L., Dalhoff, K., \& Schmiegelow, K. (2008). Pharmacogenetics influence treatment efficacy in childhood acute lymphoblastic leukemia. Journal of Paediatric Haematology \& Oncology, 30(11), 831-849.

De Bellis, M.D., Keshavan, M.S., Beers, S.R., Hall, J., Frustaci, K., Masalehdan, A., ... Boring, A.M. (2001). Sex differences in brain maturation during childhood and adolescence. Cerebral Cortex, 11, 552-557. doi: 10.1093/cercor/11.6.552

Dellani, P.R., Eder, S., Gawehn, J., Vucurevic, G., Fellgiebel, A., Muller, M.J., ...Gutjahr, P. (2008). Late structural alterations of cerebral white matter in longterm survivors of childhood leukemia. Journal of Magnetic Resonance Imaging, 27, 1250-1255. doi: $10.1002 /$ jmri.21364

Delis, D.C., Kramer, J.H., Kaplan, E., \& Ober, B.A. (1994). California Verbal Learning Test - Children's Version (CVLT-C). Pearson Assessments.

Delis, D.C., Freeland, J., Kramer, J.H., Kaplan, E. (1988). Integrating clinical assessment with cognitive neuroscience: construct validation of the California Verbal Learning Test. Journal of Consulting and Clinical Psychology, 56(1), 123-130. doi: $10.1037 / 0022-006 X .56 .1 .123$

Earle, E.A. \& Eiser, C. (2007). Children's behaviour following diagnosis of acute lymphoblastic leukemia: A qualitative longitudinal study. Clinical Child Psychology and Psychiatry, 12(2), 281-293. doi: 10.1177/1359104507075935

El-Sabagh, M.E., Ramadan, K.S., El-slam, I.M., \& Ibrahim, A.M. (2001). Antioxidants status 
in acute lymphoblastic leukemia patients. American Journal of Medicine and Medical Sciences, 1(1), 1-6. doi: 10.5923/j.ajmms.20110101.01

Espy, K.A., Moore, I.M., Kaufmann, P.M., Kramer, J.H., Matthay, K., \& Hutter, J.J. (2001). Chemotherapeutic CNS prophylaxis and neuropsychologic changes in children with acute lymphoblastic leukemia: a prospective study. Journal of Pediatric Psychology, 26(1), 1-9. doi: 10.1093/jpepsy/26.1.1

Fogarty, K., Volonino, V., Caul, J., Rongey, J., Whitman, B., O'Connor, D., \& Accardo, P. (1988). Acute leukemia: learning disabilities following CNS irradiation. Clinical Pediatrics, 27(11), 524-528. doi: 10.1177/000992288802701102

Frankland, P.W. \& Bontempi, B. (2005). The organization of recent and remote memories. Nature Reviews Neuroscience, 6, 119-130.

Gay, C.T., Bodensteiner, J.B., Nitschke, R., Sexauer, C. \& Wilson, D. (1989). Reversible treatment-related leurkoencephalopathy. Journal of Child Neurology, 4, 209-213. doi: $10.1177 / 088307388900400312$

Gluck, M.A. \& Myers, C.E. (1998). Psychobiological models of hippocampal function in learning and memory. In J. Martinez \& R. Kesner, Neurobiology of learning and memory (pp. 417-448). Joe United States of America: Academic Press.

Haddy, T.B., Mosher, R.B., \& Reaman, G.H. (2009). Late effects in long-term survivors after treatment for childhood acute leukemia. Clinical Pediatrics, 48(6), 601-608. doi: $10.1177 / 0009922809332680$

Hale, J.P. \& Lilleyman, J.S. (1991). Importance of 6-mercaptopurine dose in lymphoblastic leukemia. Archives of Disease in Childhood, 66, 462-466.

Harila, M.J., Winqvist, S., Lanning, M., Bloigu, R., \& Harila-Saari, A.H. (2009). Progressive 
neurocognitive impairment in young adult survivors of childhood acute lymphoblastic leukemia. Pediatric Blood Cancer, 53, 156-161. doi: 10.1002/pbc.21992

Heckl, S., Aschoff, A., \& Kunze, S. (2002). Radiation-induced cavernous hemangiomas of the brain: A late effect predominantly in children. Cancer, 94, 3285-3291.

Hunger, S.P., Raetz, E.A., Loh, M.L., \& Mullighan, C.G. (2011). Improving outcomes for high-risk ALL: translating new discoveries into clinical care. Pediatric Blood Cancer, 56, 984-993. doi: 10.1002/pbc.22996

Inaba, H. \& Pui, C-H. (2010). Glucocorticoid use in acute lymphoblastic leukemia. Lancet of Oncology, 11, 1096-1106. doi: 10.1016/S1470-2045(10)70114-5

Jain, N., Brouwers, P., Okcu, M.F., Cirino, P.T., \& Krull, K.R. (2009). Sex-specific attention problems in long-term survivors of pediatric acute lymphoblastic leukemia. Cancer, 115, 4238-4245. doi: $10.1002 /$ cncr.24464

Jankovic, M., Brouwers, P., Valsecchi, M.G., Van Veldhuizen, A., Huisman, J., Kamphuis, R., ... Masera, G. (1994). Association of $1800 \mathrm{cGy}$ cranial irradiation with intellectual function in children with acute lymphoblastic leukemia. The Lancet, 344, 224-7.

Jonides, J. \& Nee, D.E. (2006). Brain mechanisms of proactive interference in working memory. Neuroscience, 139, 181-193. doi: 10.1016/j.neuroscience.2005.06.042

Kadan-Lottick, N.S., Brouwers, P., Breiger, D., Kaleita, T., Dziura, J., Northrup, ... Neglia, J.P. (2009). Comparison of neurocognitive functioning in children previously randomly assigned to intrathecal methotrexate compared with triple intrathecal therapy for the treatment of childhood acute lymphoblastic leukemia. Journal of Clinical Oncology, 27, 5986-5992.doi: 10.1200/JC).2009.23.5408

Kail, R. (2002). Developmental change in proactive interference. Child Development, 73(6), 
1703-1714. doi: $10.1111 / 1467-8624.00500$

Kesner, R.P. (1998). Neurobiological views of memory. In J. Martinez \& R. Kesner (Eds.), Neurobiology of learning and memory (pp. 361-416). United States of America: Academic Press.

Khong, P.L., Leung, L.H., Fung, A.S., Fong, D.Y., Qiu, D., Kwong, D., ... Chan, G.C. (2006). White matter anisotropy in post-treatment childhood cancer survivors: Preliminary evidence of association with neurocognitive function. Journal of Clinical Oncology, 24(6), 884-890. doi: 10.1200/JCO.2005.02.4505

Krajinovic, M., Labuda, D., \& Sinnett, D. (2001). Childhood acute lymphoblastic leukemia: genetic determinants of susceptibility and disease outcome. Reviews on Environmental Health, 16(4), 263-279.

Krajinovic, M., Robaey, P., Chiasson, S., Lemieux-Blanchard, E., Rouillard, M., Primeau, M., ... Moghrabi, A. (2005). Polymorphisms of genes controlling homocysteine levels and IQ score following the treatment for childhood ALL. Pharmacogenomics, 6(3), 293-302. doi: 10.1517/14622416.6.3.293

Kramer, J.H. \& Delis, D.C. (1991). Interference effects on the California Verbal Learning Test: a construct validation study. Journal of Consulting and Clinical Psychology, 3(2), 299-302. doi: 10.1037/1040-3590.3.1.19

Krull, K.R., Khan, R.B., Ness, K.K., Ledet, D., Zhu, L., Pui, .. Morris, E.B. (2011). Symptoms of Attention-Deficit/Hyperactivity Disorder in long-term survivors of childhood leukemia. Pediatric Blood Cancer, 57, 1191-1196. doi: 10.1002/pbc.22994 Langer, T., Martus, P., Ottensmeier, H., Hertzberg, H., Beck, J.D., \& Meier, W. (2002). CNS late-effects after ALL therapy in childhood. Part III: neuropsychological performance 
in long-term survivors of childhood ALL: impairments of concentration, attention, and memory. Medical and Pediatric Oncology, 38, 320-328. doi: 10.1002/mpo.10055

Laningham, F.H., Kun, L.E., Reddick, W.E., Ogg, R.J., Morris, E.B., \& Pui, C-H. (2007). Childhood central nervous system leukemia: historical perspectives, current therapy, and acute neurological sequelae. Neuroradiology, 49(11), 873-888. doi: $10.1007 / \mathrm{s} 00234-007-0300-7$

Linabery, A.M., \& Ross, J.A. (2008). Trends in childhood cancer incidence in the U.S. (1992-2004). Cancer, 112, 416-432. doi: 10.1002/cncr.23169

Lockwood, K.A., Bell, T.S., \& Colegrove, R.W. (1999). Long-term effects of cranial radiation therapy on attention functioning in survivors of childhood leukemia. Journal of Pediatric Psychology, 24(1), 55-66. doi: 10.1093/jpepsy/24.1.55

Lofstad, G.E., Reinfjell, T., Hestad, K., \& Diseth, T. (2009). Cognitive outcome in children and adolescents treated for acute lymphoblastic leukemia with chemotherapy only. Acta Paediatrica, 98, 180-186. doi: 10.1111/j.1651-2227.2008.01055.x

Lupien, S. J., Gillin, C. J., \& Hauger, R. L. (1999). Working memory is more sensitive than declarative memory to the acute effects of corticosteroids: a dose-response study in humans. Behavioural Neuroscience, 113, 420-430.

Lustig, C., May, C.P., \& Hasher, L. (2001). Working memory span and the role of proactive interference. Journal of Experimental Psychology: General, 130(2), 199-207. doi: 10.1037/0096-3445.130.2.199

Mandrell, B.N. (2009). The genetic profile and monitoring of acute lymphoblastic leukemia in children and adolescents. Journal of Pediatric Nursing, 24(3), 173-178. 
Marcoux, S., Robaey, P., Krajinovic, M., Moghrabi, A., \& Laverdière, C. (2012).

Predictive factors of internalized and externalized behavioral problems in children treated for acute lymphoblastic leukemia. Pediatric Blood Cancer, 58(6), 1-7. doi: $10.1002 / \mathrm{pbc} .24079$

McClelland, J.L., McNaughton, B.L., \& O'Reilly, R.C. (1995). Why there are complementary learning systems in the hippocampus and neocortex: insights from the successes and failures of connectionist models of learning an memory. Psychology Review, 102, 419-457.

Mennes, M., Stiers, P., Vandenbussche, E., Vercruysse, G., Uyttebroeck, A., De Meyer, G., \& Van Gool, S.W. (2004). Attention and information processing in survivors of childhood acute lymphoblastic leukemia treated with chemotherapy only. Pediatric Blood Cancer, 43, 780-787. doi: 10.1002/pbc.20147

Mitchell, C.D., Richards, S.M., Kinsey, S.E., Lilleyman, J., Vora, A., \& Eden, T.O. (2005). Benefit of dexamethasone compared with prednisone for childhood acute lymphoblastic leukemia: results of the UK Medical Research Council ALL97 randomized trial. British Journal of Haematology, 129(6), 734-745. doi: $10.1111 / \mathrm{j} .1365-2141.2005 .05509 . x$

Monje, M., Thomason, M.E., Rigolo, L., Wang, Y., Waber, D.P., ...Golby, A.J. (2012). Functional and structural differences in the hippocampus associated with memory deficits in adult survivors of acute lymphoblastic leukemia. Pediatric Blood Cancer. Advance online publication. doi: $10.1002 / \mathrm{pbc} .24263$

Montour-Proulx, I., Kuehn, S.M., Keene, D.L., Barrowman, N.J., Hsu, E., Matzinger, M-A., ... Halton, J. (2005). Cognitive changes in children treated for acute lymphoblastic 
leukemia with chemotherapy only according to the Pediatric Oncology Group 9605 protocol. Journal of Child Neurology, 20, 129-133.

Mrakotsky, C.M., Silverman, L.B., Dahlberg, S.E., Alyman, M.C.A., Sands, S.A.,Queally, J.T., ... Waber, D.P. (2011). Neurobehavioural side effects of corticosteroids during active treatment for acute lymphoblastic leukemia in children are age-dependent: report from Dana-Farber Cancer Institute ALL consortium protocol 00-01. Pediatric Blood Cancer, 57, 492-498. doi: 10.1002/pbc.23060

Ng, S.M., Lin, H.P., Ariffin, W.A., Zainab, A.K., Lam, S.K., \& Chan, L.L. (2000). Age, sex, haemoglobin level, and white cell count at diagnosis are important prognostic factors in children with acute lymphoblastic leukemia treated with BFM-type protocol. Journal of Tropical Pediatrics, 46, 338-343.

Estby, Y., Tamnes, C.K., Fjell, A.M., \& Walhovd, K.B. (2011). Morphometry and connectivity of the fronto-parietal verbal working memory network in development. Neuropsychologia, 49, 3854-3862. doi: 10.1016/j.neuropsychologia.2011.10001

Packan, D.R. \& Sapolsky, R.M. (1990). Glucocorticoid endangerment of the hippocampus: tissue, steroid, and receptor specificity. Neuroendocrinology, 51, 613-618. doi: $10.1159 / 000125400$

Peterson, C.C., Johnson, C.E., Ramirez, L.Y., Huestis, S., Pai, A.L.H., Demaree, H.A., \& Drotar, D. (2008). A meta-analysis of the neuropsychological sequelae of chemotherapy-only treatment for pediatric acute lymphoblastic leukemia. Pediatric Blood Cancer, 51, 99-104. doi: 10.1002/pbc.21544

Plasschaert, S.L.A., Kamps, W.A., Vellenga, E., de Vries, E.G.E., \& de Bont, E.S. (2004). Prognosis in childhood and adult acute lymphoblastic leukemia: a 
question of maturation?. Cancer Treatment Reviews, 30, 37-51. doi: $10.1016 / \mathrm{S} 0305-7372(03) 00140-3$

Porto, L., Preibisch, C., Hattingen, E., Bartels, M., Lehrnbecher, T., Dewitz, R., ... \& Kieslich, M. (2008). Voxel-based morphometry and diffusion-tensor MR imaging of the brain in long-term survivors of childhood leukemia. European Journal of Radiology, 18, 2691-2700.

Precourt, S., Robaey, P., Lamothe, I., Lassonde, M., Sauerwin, H.C., \& Moghrabi, A. (2002). Verbal cognitive functioning and learning in girls treated for acute lymphoblastic leukemia by chemotherapy with or without cranial irradiation. Developmental Neuropsychology, 21(2), 173-195. Doi: 10.1207/S15326942DN2102_4

Pui, C-H., Relling, M.V., \& Evans, W.E. (2003). Role of pharmacogenomics and pharmacodynamics in the treatment of acute lymphoblastic leukemia. Best Practice \& Research Clinical Haematology, 15(4), 741-756. doi: 10.1053/beha.2002.0225

Pui, C. \& Evans, W.E. (2006). Treatment of acute lymphoblastic leukemia. New England Journal of Medicine, 354, 166-178.

Raudenbush, S.W., Bryk, A.S., Cheong, Y.F., Congdon, Jr., R.T., \& du Toit, M. (2011). HLM 7: Hierarchical linear and nonlinear modeling. United States of America: Scientific Software International.

Reddick, W.E., Glass, J.O., Helton, K.J., Langston, J.W., Li, C.S., \& Pui, C.H. (2005). A quantitative MR imaging assessment of leukoencephalopathy in children treated for acute lymphoblastic leukemia without irradiation. American Journal of Neuroradiology, 26, 2371-2377. 
Ries, L.A.G., Melbert, D., Krapcho, M., Stinchcomb, D.G., Howlader, N., Horner, M.J., ... Edwards, B.K. (2008). SEER Cancer Statistics Review, 1975-2005. Bethesda, MD: National Cancer Institute. http://seer.cancer.gov/csr/1975_2005/, based on November 2007 SEER data submission, posted to the SEER Web site, 2008.

Robinson, K.E., Livesay, K.L., Campbell L.K. Scaduto, M., Cannistraci, C.J., ... Compas, B.E. (2010). Working memory in survivors of childhood acute lymphocytic leukemia: functional neuroimaging analyses. Pediatric Blood Cancer, 54(4), 585590.

Rodgers, J., Horrocks, J., Britton, P.G., \& Kernahan, J. (2003). Attentional ability among survivors of leukemia. Archives of Disease in Childhood, 80, 318-323.

Rodgers, J., Britton, P.G., Morris, R.G., Kernahan, J., \& Craft, A.W. (1992). Memory after treatment for acute lymphoblastic leukemia. Archives of Disease in Childhood, 67, 266-268.

Ronghe, M., Burke, G.A.A., Lowis, S.P., \& Estlin, E.J. (2001). Remission induction therapy for childhood acute lymphoblastic leukemia: clinical and cellular pharmacology of vincristine, corticosteroids, L-asparaginase and anthracyclines. Cancer Treatment Reviews, 27, 327-337. doi: 10.1053/ctrv.2001.0243

Roman, D.D. \& Sperduto, P.W. (1995). Neuropsychological effects of cranial radiation : Current knowledge and future directions. International Journal of Radiation Oncology Biology Physics, 31(4), 983-998. doi: 10.1016/0360-3016(94)00550-8

Rosen, V.M. \& Engle, R.W. (1998). Working memory capacity and suppression. Journal of Memory and Language, 39, 418-436.

Sallan, S.E. (2006). Myths and lessons from the adult/pediatric interface in acute 
lymphoblastic leukemia in adults and children. Hematology 2006, 128-132. doi:

10.1182/asheducation-2006.1.133

Sapolsky, R.M. (2000). Glucocorticoids and hippocampal atrophy in neuropsychiatric disorders. Archives of General Psychiatry, 57, 925-933.

Schafter, J.L. \& Olsen, M.K. (1998). Multiple imputation for multivariate missing-data problems: A data analyst's perspective. Multivariate Behavioural Research, 33(4), $545-571$.

Schatz, J., Kramer, J.H., Ablin, A., \& Matthay, K.K. (2000). Processing speed, working memory, and IQ: a developmental model of cognitive deficits following cranial radiation therapy. Neuropsychology, $14(2), 189-200$. doi: 10.1037//08944105.14.2.189

Schatz, J., Kramer, J.H., Ablin, A.R., \& Matthay, K.K. (2004). Visual attention in long-term survivors of leukemia receiving cranial radiation therapy. Journal of the International Neuropsychological Society, 10, 211-220. doi: 10.1017/S1355617704102075

Schmiegelow, K., Glomstein, A., Kristinsson, J., Salmi, T., Schroder, H., \& Bjork, O. (1997). Impact of morning versus evening schedule for oral methotrexate and 6mercaptopurine on relapse risk for children with acute lymphoblastic leukemia. Nordic Society for Pediatric Hematology and Oncology (NOPHO). Journal of Pediatric Hematology \& Oncology, 19, 102-109.

Schmithorst, V.J. (2009). Developmental sex differences in the relation of neuroanatomical connectivity to intelligence. Intelligence, 37, 164-173. doi: 10.1016/j.intell.2008.07.001 
Schmithorst, V.J., Wilke, M., Dardzinski, B.J., \& Holland, S.K. (2005). Cognitive functions correlate with white matter architecture in a normal pediatric population: A diffusion tensor MRI study. Human Brain Mapping, 26, 139-147. doi: $10.1002 / \mathrm{hbm} .20149$

Squire, L.R. \& Alvarez, P. (1995). Retrograde amnesia and memory consolidation: a neurobiological perspective. Current Opinions in Neurobiology, 5, 169-177.

Spiegler, B.J., Kennedy, K., Maze, R., Greenberg, M.L., Weitzman, S., Hitzler, J.K., \& Nathan, P.C. (2006). Comparison of long-term neurocognitive outcomes in young children with acute lymphoblastic leukemia treated with cranial radiation or high-dose or very high-dose intravenous methotrexate. Journal of Clinical Oncology, 24, 38583864.

Stanulla, M., \& Schrappe, M. (2009). Treatment of childhood acute lymphoblastic leukemia. Seminars in Hematology, 46(1), 52-63. doi:

10.1053/j.seminhematol.2008.09.007

Stuart, F.A., Segal, T.Y., \& Keady, S. (2005). Adverse psychological effects of corticosteroids in children and adolescents. Archives of Disease in Childhood, 90, 500-506. doi: 10.1136/adc.2003.041541

Takeuchi, H., Taki, Y., Sassa, Y., Hashizume, H., Sekiguchi, A., Fukushima, A., \& Kawashima, R. (2011). Verbal working memory performance correlation with regional white matter structures in the frontoparietal regions. Neuropsychologia, 49, 3466-3473. doi: 10.1016/j.neuropsychologia.2011.08.022 
Vitaro, F., Tremblay, R.E., \&Gagnon, C. (1992). Adversité familiale et troubles du comportement au début de la période de frequentation scolaire. Revue Canadienne de Santé Mentale Communautaire, 11(1), 45-62.

Waber, D.P., Bernstein, J.H., Kammerer, B.L., Tarbell, N.J., Sallan, S.E. (1992). Neuropsychological diagnostic profiles of children who received CNS treatment for acute lymphoblastic leukemia: the systemic approach to assessment. Developmental Neuropsychology, 8(1), 1-28. doi: 10.1080/87565649209540512

Waber, D.P., Gioia, G., Paccia, J., Sherman, B., Dinklage, D., Sollee, N., ... Sallan, S.E. (1990). Sex differences in cognitive processing in children treated with CNS prophylaxis for acute lymphoblastic leukemia. Journal of Pediatric Psychology, 15(1), 105-122. doi: 10.1093/jpesy/15.1.105

Waber, D.P., Urion, D.K., Tarbell, N.J., Niemeyer, C. (1990). Late effects of central nervous system treatment of acute lymphoblastic leukemia in childhood are sex-dependent. Developmental Medicine and Child Neurology, 32(3), 238-248.

Witigen, B.J., Zhou, M., Cai, Yi., Balaji, J., Guzman Karlsson, M., Parivash, S.N., .... Silva, A.J. (2010). The hippocampus plays a selective role in the retrieval of detailed contextual memories. Current Biology, 20, 1-9. doi: 10.1016/j.cub.2010.06.068 Whitney, P., Arnett P.A., Driver, A. \& Budd, D. (2001). Measuring central executive functioning: what's in a reading span?. Brain and Cognition, 45, 1-14. 


\section{Appendix A}

\section{Study II: Research Ethics Board Approval}

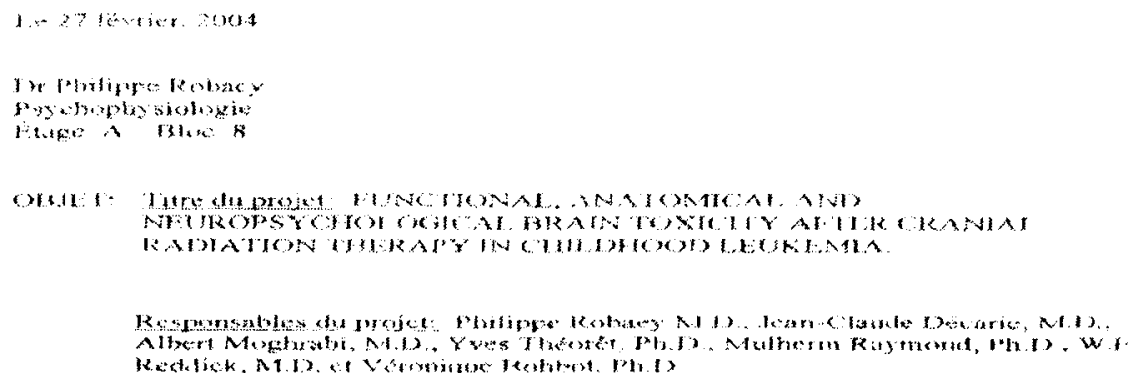

Wher Deriteut.

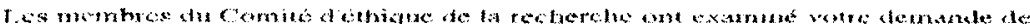

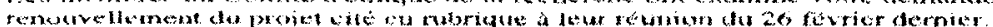

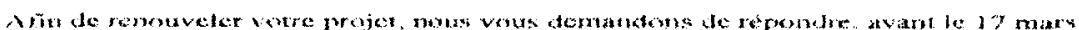

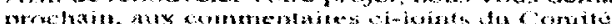

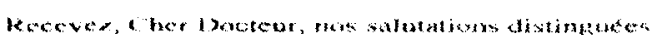

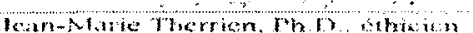

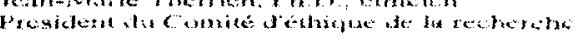

JMic

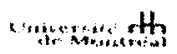


Appendix B

Syntax Used to Calculate Proactive Inference, Retroactive Interference, and Learning Slope

*****To compute standardized proactive interference*****

DATASET ACTIVATE DataSet1.

COMPUTE proactivez $1=(\operatorname{cvbtot} 1 n)-(\operatorname{cv} 1$ tot $1 \mathrm{n})$.

EXECUTE.

DATASET ACTIVATE DataSet1.

COMPUTE proactivez3=(cvbtot3n) $-(\operatorname{cvltot} 3 n)$.

EXECUTE.

DATASET ACTIVATE DataSet1.

COMPUTE proactivez4=(cvbtot4n) $-(\operatorname{cvl} 1$ tot $4 n)$.

EXECUTE.

DATASET ACTIVATE DataSet1.

COMPUTE proactivez5 $=(\operatorname{cvbtot} 5 n)-(\operatorname{cvltot} 5 n)$.

EXECUTE.

DATASET ACTIVATE DataSet1.

COMPUTE proactivez6 $=(\operatorname{cvbtot} 6 n)-($ cv1tot6n $)$.

EXECUTE.

$* * * * *$ To compute standardized retroactive interference

DATASET ACTIVATE DataSet1.

COMPUTE retroactivez $1=($ cvatot $1 n)-(\operatorname{cv} 5$ tot $1 n)$.

EXECUTE.

DATASET ACTIVATE DataSet1.

COMPUTE retroactivez3 $=(\operatorname{cvatot} 3 n)-(\operatorname{cv} 5$ tot $3 n)$.

EXECUTE.

DATASET ACTIVATE DataSet1.

COMPUTE retroactivez4 $=(\operatorname{cvatot} 4 n)-(\operatorname{cv} 5$ tot $4 n)$.

EXECUTE.

DATASET ACTIVATE DataSet1.

COMPUTE retroactivez5 $=($ cvatot $4 n)-(\operatorname{cv} 5$ tot $5 n)$.

EXECUTE.

DATASET ACTIVATE DataSet1.

COMPUTE retroactivez6 $=($ cvatot6n $)-($ cv5tot6n $)$.

EXECUTE. 
*********To compute learning slope $e^{* * * * * * * *}$

DATASET ACTIVATE DataSetl.

COMPUTE

learningslope $1=(((1 * \operatorname{cv} 1$ tot 1$)+(2 * \operatorname{cv} 2$ tot 1$)+(3 * \operatorname{cv} 3$ tot 1$)+(4 * \operatorname{cv} 4$ tot 1$)+(5 * \operatorname{cv} 5$ tot 1$)) / 5) / 2$.

EXECUTE.

DATASET ACTIVATE DataSet1.

COMPUTE

learningslope $3=(((1 * \operatorname{cv} 1$ tot 3$)+(2 * \operatorname{cv} 2 \operatorname{tot} 3)+(3 * \operatorname{cv} 3$ tot 3$)+(4 * \operatorname{cv} 4$ tot 3$)+(5 * \operatorname{cv} 5$ tot 3$)) / 5) / 2$.

EXECUTE.

DATASET ACTIVATE DataSet1.

COMPUTE

learningslope $4=(((1 * \operatorname{cv} 1$ tot 4$)+(2 * \operatorname{cv} 2$ tot 4$)+(3 * \operatorname{cv} 3 \operatorname{tot} 4)+(4 * \operatorname{cv} 4$ tot 4$)+(5 * \operatorname{cv} 5$ tot 4$)) / 5) / 2$.

EXECUTE.

DATASET ACTIVATE DataSet1.

COMPUTE

learningslope $5=(((1 * \operatorname{cv} 1$ tot 5$)+(2 * \operatorname{cv} 2$ tot 5$)+(3 * \operatorname{cv} 3$ tot 5$)+(4 * \operatorname{cv} 4$ tot 5$)+(5 * \operatorname{cv} 5$ tot 5$)) / 5) / 2$.

EXECUTE.

DATASET ACTIVATE DataSet1.

COMPUTE

learningslope $6=(((1 * \operatorname{cv} 1$ tot 6$)+(2 * \operatorname{cv} 2$ tot 6$)+(3 * \operatorname{cv} 3$ tot 6$)+(4 * \operatorname{cv} 4$ tot 6$)+(5 * \operatorname{cv} 5$ tot 6$)) / 5) / 2$.

EXECUTE.

Where:

proactivez $n=$ standardized score for proactive interference at Time $n$

$\operatorname{cvbtot} n n=$ age-corrected score for total number of words recalled on List B at Time

$n$

cvltot $n n=$ age-corrected score for total number of words recalled on List A Trial 1 at Time $n$

retroactivez $n=$ standardized score for retroactive interference at Time $n$

cvatot $n \mathrm{n}=$ age-corrected score for total number of words recalled on Short Delay List A at Time $n$

cv5tot $n n=$ age-corrected score for total number of words recalled on List A Trial 5 at Time $n$

learningslope $n=$ learning slope at Time $n$

cvltot $n=$ number of words recalled on List A Trial 1 at Time $n$ $\mathrm{cv} 2$ tot $n=$ number of words recalled on List A Trial 2 at Time $n$ $\mathrm{cv} 3$ tot $n=$ number of words recalled on List A Trial 3 at Time $n$ cv4tot $n=$ number of words recalled on List A Trial 4 at Time $n$ $\operatorname{cv} 5$ tot $n=$ number of words recalled on List A Trial 5 at Time $n$ 
Appendix C

Study II: Assessment of Linearity

Proactive Interference

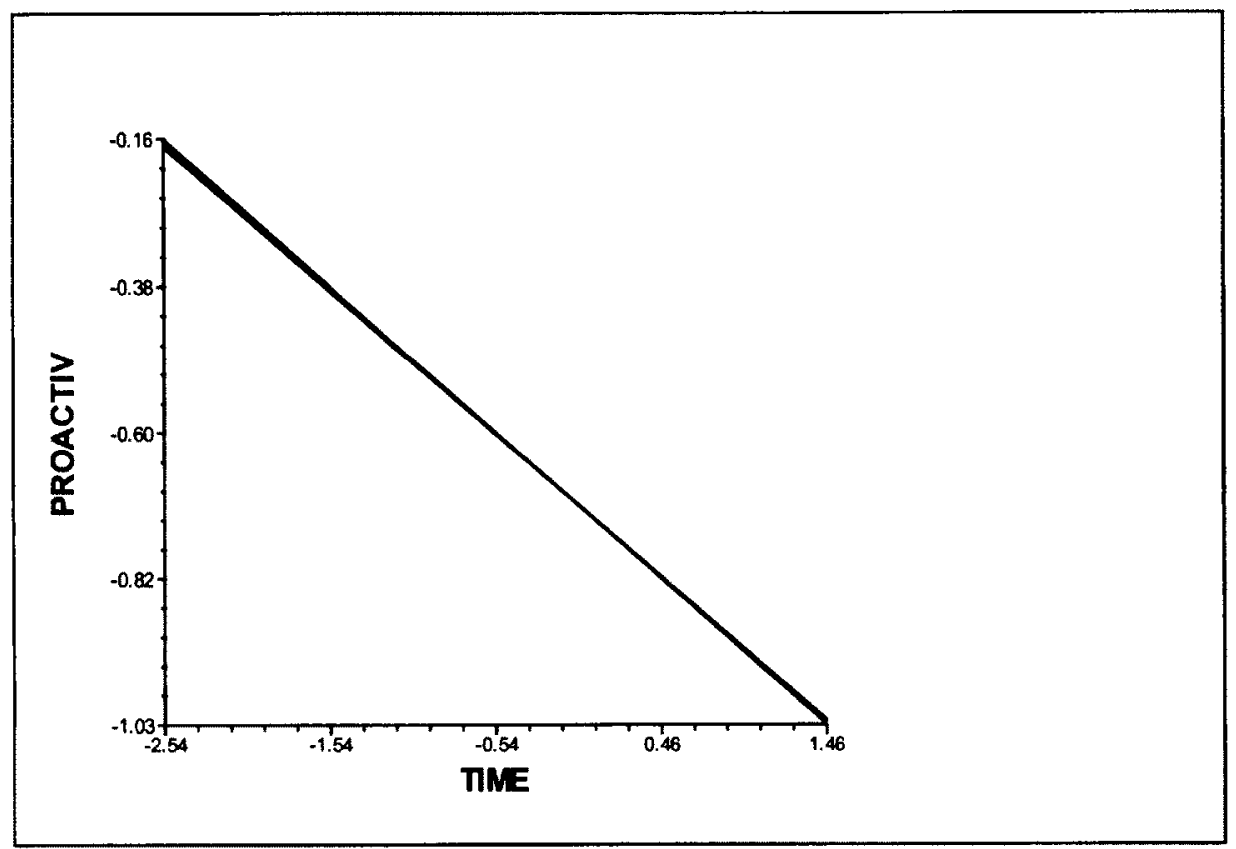

Learning Slope

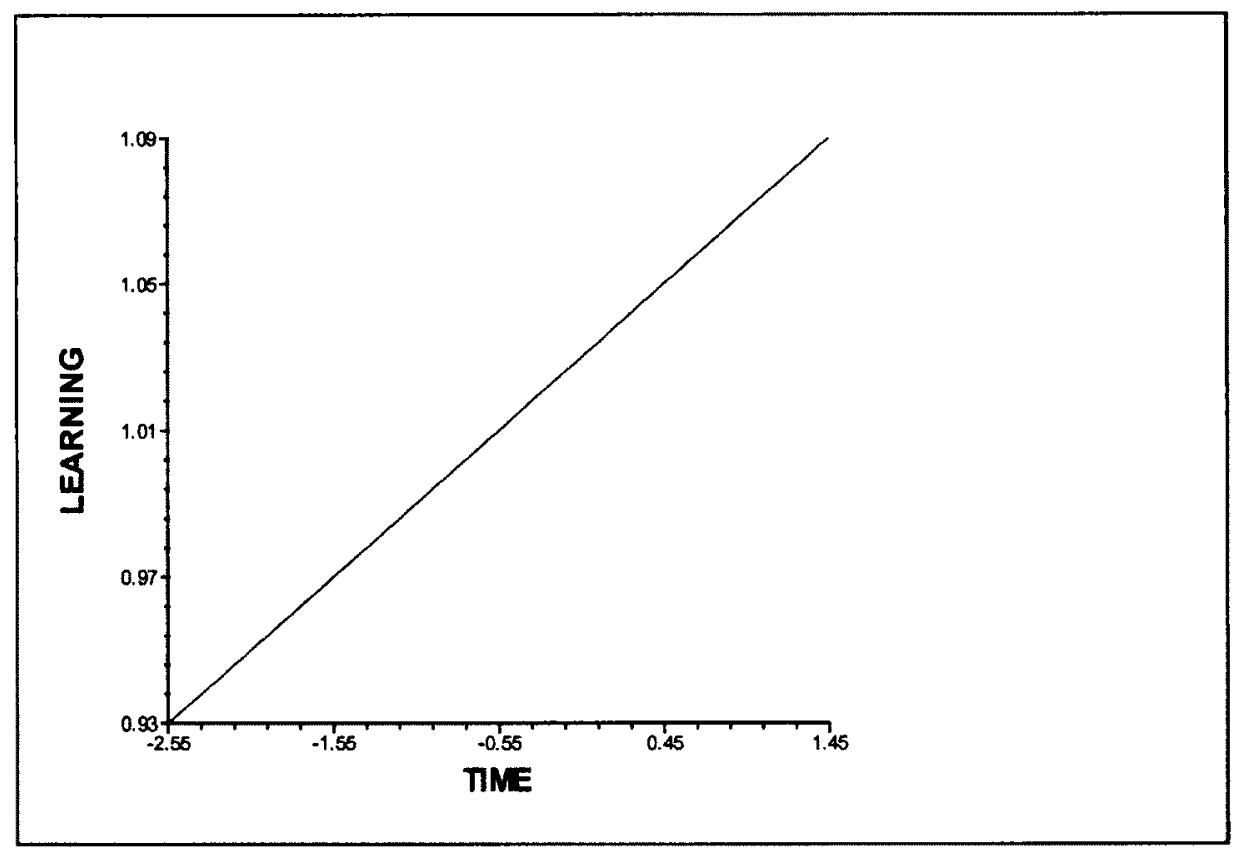

List A Free Recall 


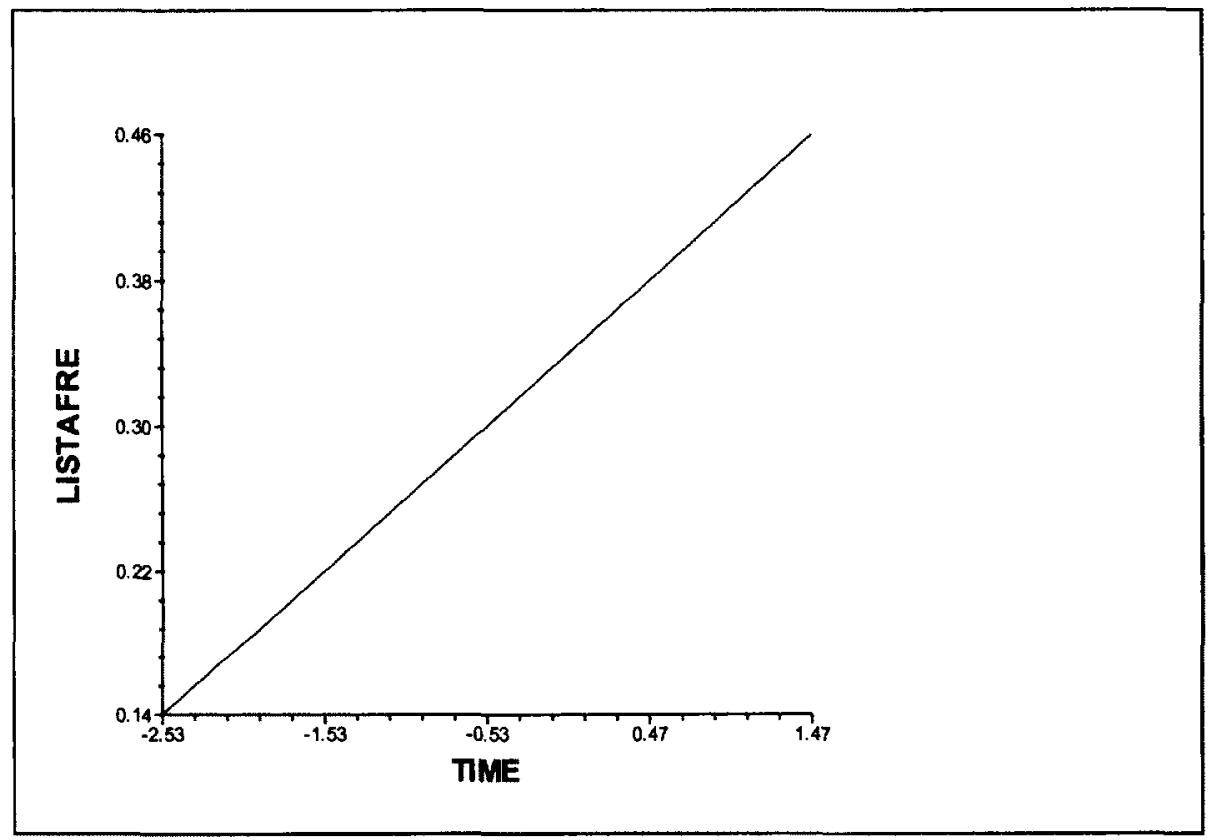

List B Recall

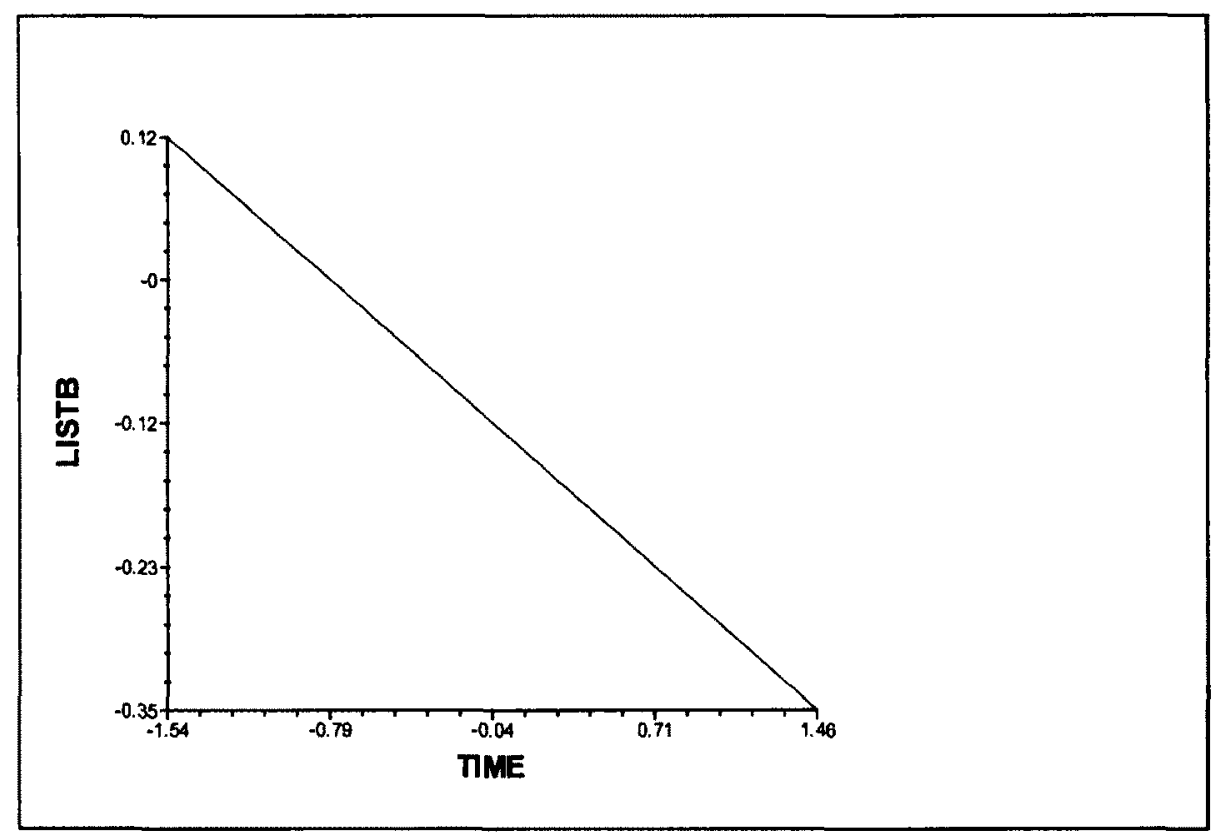

Total Number of Words Recalled from Trial 1 to Trial 5 


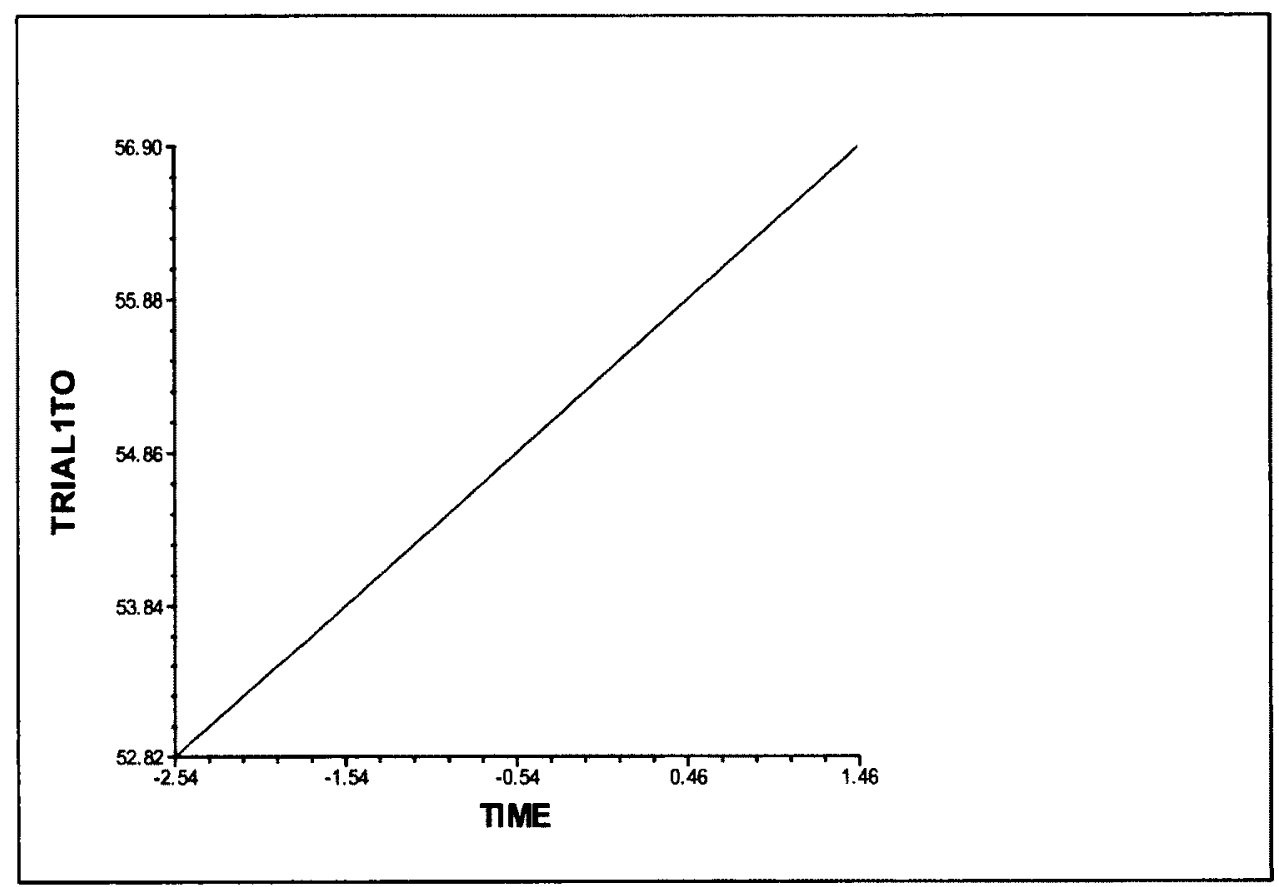


Appendix D

Study II: Assessment of Normality

Learning Slope

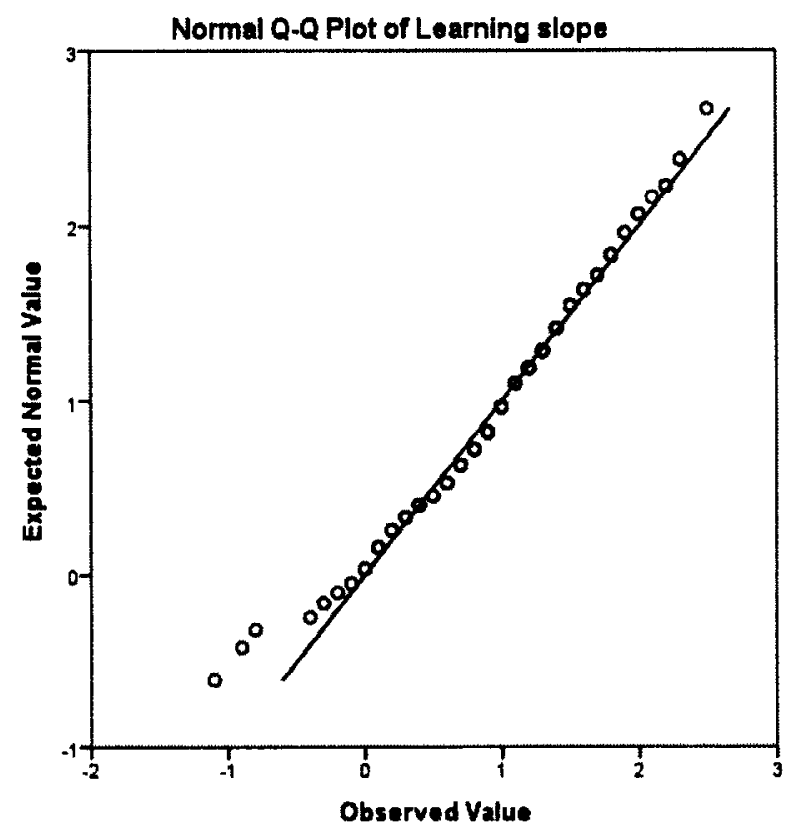

List A Free Recall

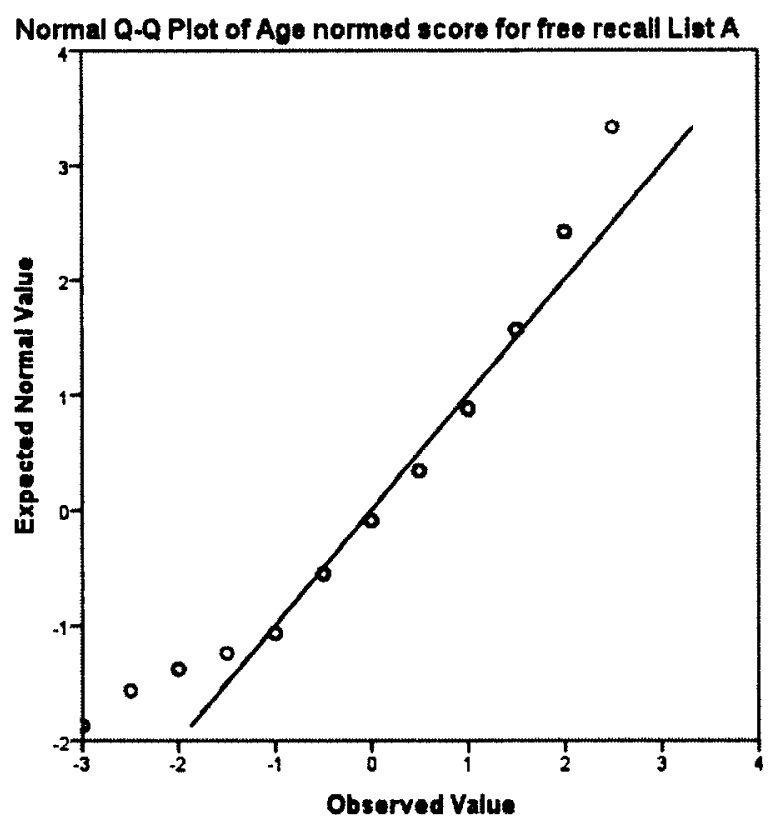


List B Recall

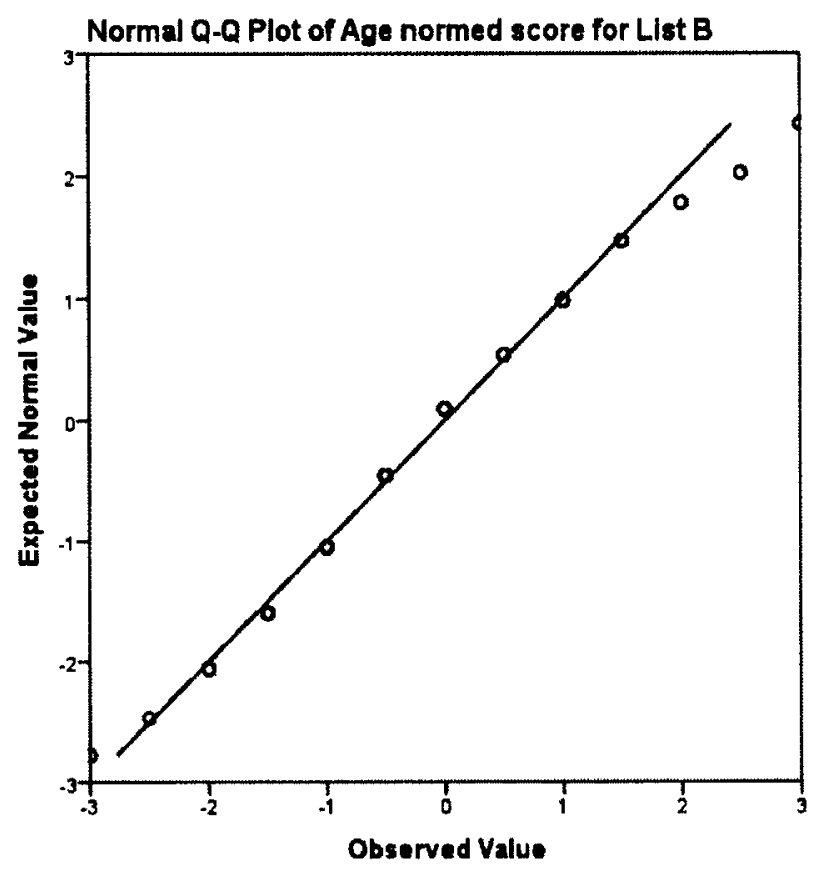

Proactive Interference

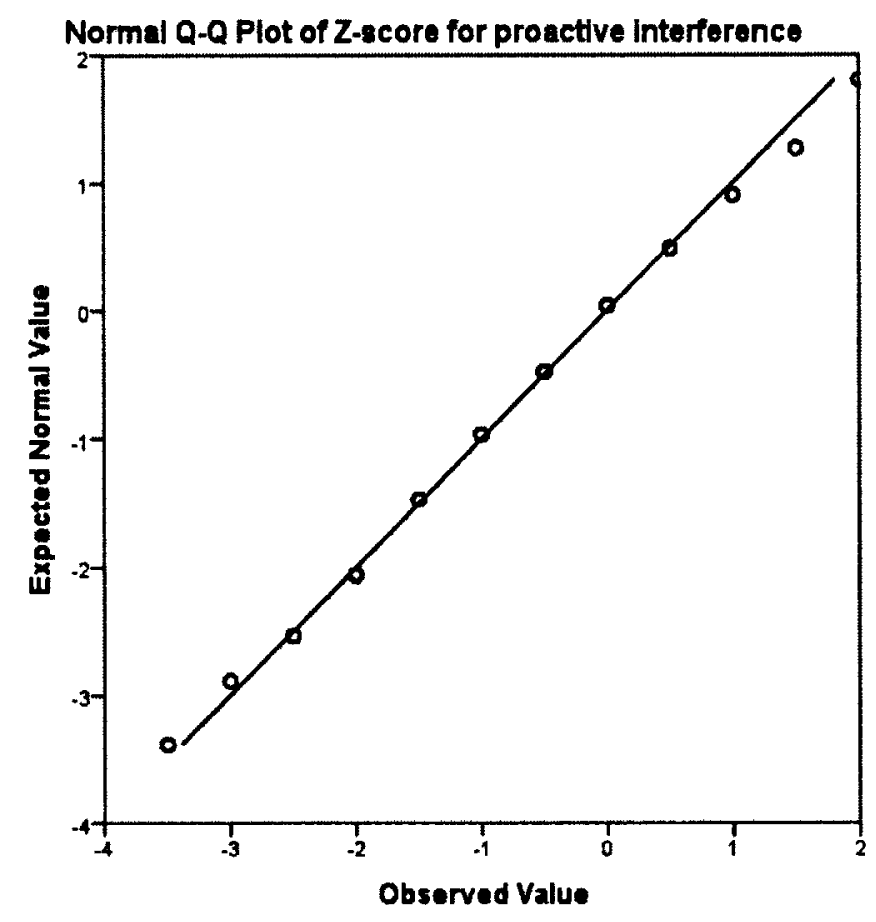


Total Number of Words Recalled From Trial 1 to Trial 5

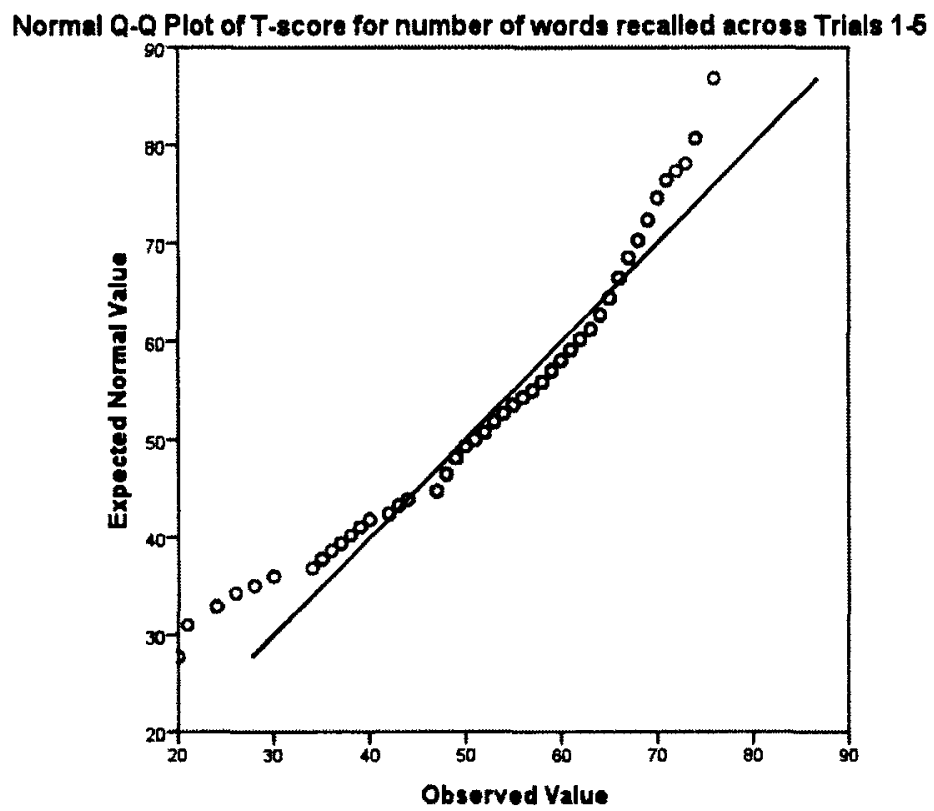


Appendix E

Study II: Testing of Heteroscedasticity

HLM 6 Output

Learning Slope

Test of homogeneity of level-1 variance

$\chi^{2}$ statistic $=5.32900$

degrees of freedom $=35$

$p$-value $=>.500$

List A Free Recall

Test of homogeneity of level-1 variance

$\chi^{2}$ statistic $=17.00628$

degrees of freedom $=49$

$p$-value $=>.500$

List B Recall

Test of homogeneity of level-1 variance

$\chi^{2}$ statistic $=8.69189$

degrees of freedom $=36$

$p$-value $=>.500$

Proactive Interference

Test of homogeneity of level-1 variance 
$\chi^{2}$ statistic $=10.04322$

degrees of freedom $=25$

$p$-value $=>.500$

Total Number of Words Recalled from Trial 1 to Trial 5

Test of homogeneity of level-1 variance

$\chi^{2}$ statistic $=92.28662$

degrees of freedom $=50$

$p$-value $=0.000$ 